\title{
Numerical Simulation of the Effect of Different Number Leading Edge Winglets on the Fan-Wing Aerodynamic Characteristics
}

\author{
Du Siliang $\mathbb{D}^{1,2,3}$ Zhao Qijun $\mathbb{D}^{1}{ }^{1}$ and Tang Zhengfei $\mathbb{D}^{1}$ \\ ${ }^{1}$ National Key Laboratory of Rotorcraft Aeromechanics, Nanjing University of Aeronautics and Astronautics, Nanjing 210016, China \\ ${ }^{2}$ Faculty of Mechanical \& Material Engineering, Huaiyin Institute of Technology, Huai'an 223003, China \\ ${ }^{3}$ Rotor Aerodynamics Key Laboratory, China Aerodynamics Research and Development Center, Mianyang Sichuan 621000, China
}

Correspondence should be addressed to Du Siliang; kjofchina@qq.com and Zhao Qijun; zhaoqijun@nuaa.edu.cn

Received 25 August 2019; Revised 1 January 2020; Accepted 18 January 2020; Published 11 February 2020

Academic Editor: William W. Liou

Copyright () $2020 \mathrm{Du}$ Siliang et al. This is an open access article distributed under the Creative Commons Attribution License, which permits unrestricted use, distribution, and reproduction in any medium, provided the original work is properly cited.

The generation of lift and thrust mainly depends on the formation of low-pressure vortices above the arc groove on the leading edge of the Fan-wing, which makes the lift and thrust have a strong coupling relationship. How to decouple and control the lift and thrust is the key to further engineering application of the Fan-wing. Normally, the geometric parameters of the Fan-wing airfoil were determined; the leading edge opening angle has the greatest influence on the aerodynamic performance. Therefore, the method of installing leading edge winglets on the leading edge of a base Fan-wing airfoil was considered to change the opening angle of the leading edge of the Fan-wing. Through numerical simulation, the effects of single, double, and triple leading edge winglets on lift and thrust of the Fan-wing at different installation angles, inflow velocities, and angles of attack were compared and analyzed. The results show that by controlling the angle of the leading edge winglet, not only the lift and thrust of the fan can be improved but also the strength and position of the low-pressure vortices can be controlled, so as to meet the active control requirements of the aerodynamic moment of the Fan-wing, and then the attitude of the Fan-wing aircraft can be controlled.

\section{Introduction}

Fan-wing concept with distributed propulsion is described as a simple, stable, and very efficient high lift aircraft wing. There is a crossflow fan with infinitely variable speed powered by the engine at the leading edge of each wing. The crossflow fan pulls the air in from the front and accelerates the air over the trailing edge of the wing. Therefore, Fan-wing accelerates a large volume of air and produces lift and thrust simultaneously. This kind of distributed lift and thrust of Fan-wing has higher efficiency than that gained by improving the bypass ratio of the gas turbine engine currently. The advantages of Fan-wing aircraft compared to the conventional aircraft are short take-off and landing (STOL) at the low forward speed, no stall, and high power load. Recently, the investigations on the Fan-wing technology integrated into airfoils showed the high lift potential of the embedded propulsion system and moved the research from experimentation to prototyping. Several experimental programs have been carried out to demonstrate the Fan-wing concept, including the works of Kogler [1], Peebles [2], Seyfang [3, 4], Foreshaw [5], Bayindir and Guillermo [6], Askari et al. [7-10], Duddempudi et al. [11], and Dang et al. [12, 13]. They have carried out experimental and numerical simulation analysis on the aerodynamic characteristics of the Fan-wing. It was proved that the geometric design parameters of the Fan-wing airfoil and crossflow fan blade have a great influence on the lift coefficient, thrust coefficient, and power load characteristics. It was also revealed that most of the energy for maintaining lift force of the Fan-wing comes from the lowpressure vortices formed inside the crossflow fan. Siliang's research team of the Nanjing University of Aeronautics and Astronautics [14-19] carried out the research on Fan-wing $\mathrm{UAV}$, which was the implementation team of the major exploration project of demonstration and verification of the Fan-wing aircraft in China. By means of theoretical analysis, numerical simulation, experimental verification, and flight demonstration, the source of lift thrust of the Fan-wing was 


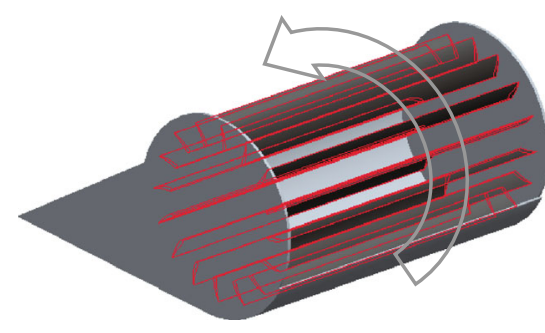

(a) CATIA model of Fan-wing

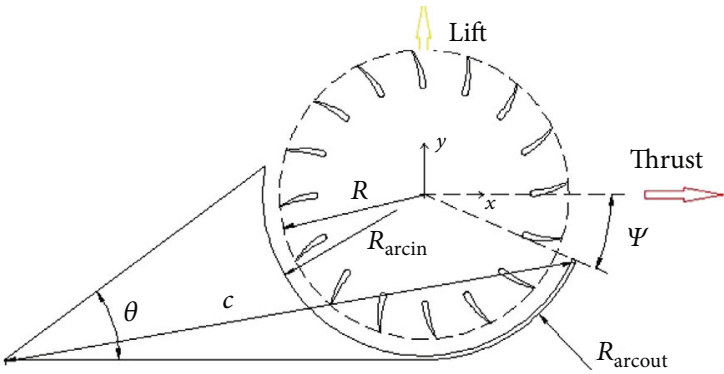

(c) Definition of airfoil parameters

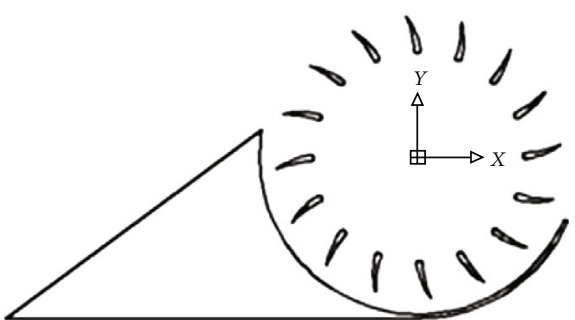

(b) Two-dimensional airfoil

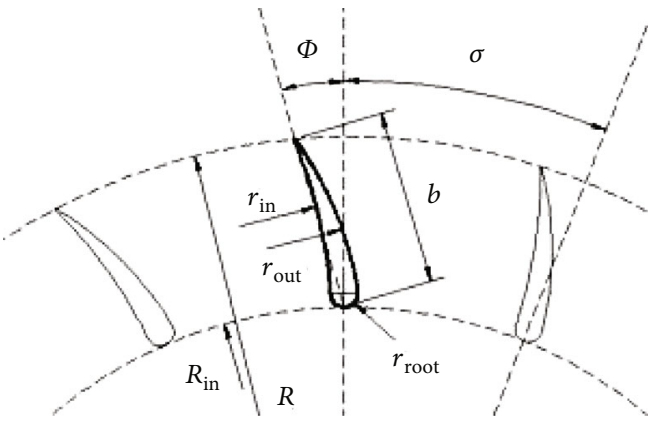

(d) Definition of blade parameters

Figure 1: Fan-wing parameters.

studied in detail and a set of structural design methods for the fan airfoil and crossflow fan are obtained.

Based on the research of the above scholars, it can be found that the leading edge opening angle was the biggest factor affecting the lift and thrust of the Fan-wing after the size of the wing airfoil and crossflow fan was determined. Within a certain opening angle, the leading edge opening angle increases, the lift and thrust increase, and the thrust increase was obvious. This was similar to the effect of the intake port on engine thrust, and the simplest way to control the opening angle of the Fan-wing was to install the leading edge winglet, which can change the opening angle of the leading edge by controlling the deflection angle of the leading edge winglet, so as to improve the aerodynamic characteristics of the Fan-wing. In this study, the numerical simulation will be used to investigate the aerodynamic control effect of the leading edge wing on the Fan-wing.

\section{Geometric Model and Numerical Method of Fa-Wing}

2.1. Definition of Geometric Model of Datum Fan-Wing. In order to reduce the research variables, it was assumed that the section along the extension direction of the Fan-wing was unchanged. Therefore, the two-dimensional airfoil section of the Fan-wing was analyzed in this paper. The tripledimensional model and two-dimensional section model established by CATIA software are shown in Figures 1(a) and 1(b). The main parameters of the airfoil and blade were defined in Figures 1(c) and 1(d) and Tables 1 and 2.

2.2. Design of Leading Edge Winglet. According to the calculation and analysis in reference [19], the leading edge opening angle, which has a great influence on the lift and thrust of the Fan-wing, was between 25 and 55 degrees. Considering
Table 1: Main parameters of Fan-wing airfoil.

\begin{tabular}{lc}
\hline Main airfoil parameters & Value \\
\hline Radius of crossflow fan $R(\mathrm{~mm})$ & 150 \\
Inner radius of semicircular cavity $R_{\text {arcin }}(\mathrm{mm})$ & 155 \\
Outer radius of semicircular cavity $R_{\text {arcout }}(\mathrm{mm})$ & 160 \\
Chord $c(\mathrm{~mm})$ & 561 \\
Trailing angle $\theta\left(^{\circ}\right)$ & 36.5 \\
Leading edge opening angle $\psi\left({ }^{\circ}\right)$ & 24 \\
\hline
\end{tabular}

TABLE 2: Main parameters of cross-flow fan blade.

\begin{tabular}{lc}
\hline Main blade parameters & Value \\
\hline Blade width $b(\mathrm{~mm})$ & 36 \\
Outer radius of crossflow fan $R(\mathrm{~mm})$ & 150 \\
Inner radius of crossflow fan $R_{\text {in }}(\mathrm{mm})$ & 98 \\
Blade outer arc radius $r_{\text {out }}(\mathrm{mm})$ & 96 \\
Blade inner arc radius $r_{\text {in }}(\mathrm{mm})$ & 68 \\
Blade root arc radius $r_{\text {root }}(\mathrm{mm})$ & 3 \\
Blade installation angle $\phi\left({ }^{\circ}\right)$ & 18 \\
Contiguous blade angle $\sigma\left({ }^{\circ}\right)$ & 22.5 \\
\hline
\end{tabular}

the reliability of the structure, a maximum of triple leading edge winglets were adopted for analysis (Figures $2(a)-2(c)$ ). The design of each winglet can control the opening angle of $10^{\circ}$ and can rotate along their respective central axis of rotation. The angle of tangent between the chord of each winglet and the arc of the leading edge arc groove of the Fan-wing was defined as 0 degree and the counter-clockwise rotation 


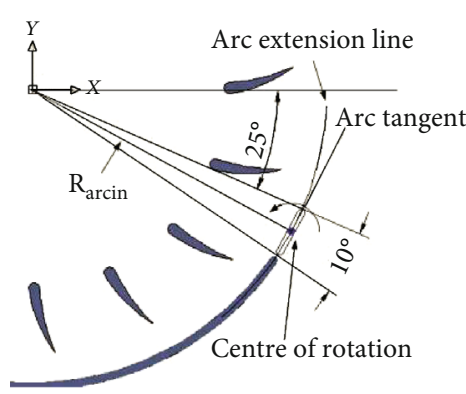

(a) Single leading edge winglet

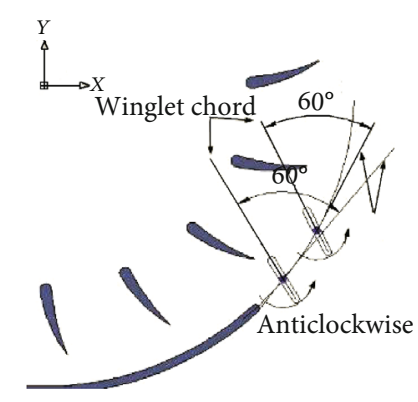

(b) Double leading edge winglets

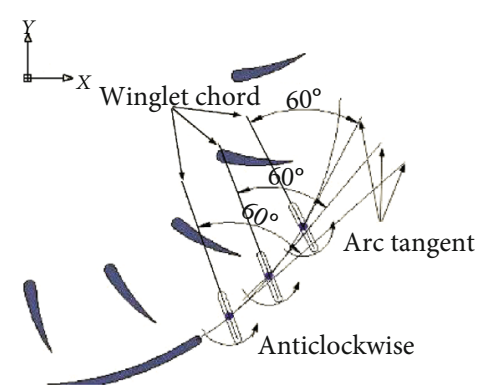

(c) Triple leading edge winglets

Figure 2: Design of leading edge winglet of Fan-wing.

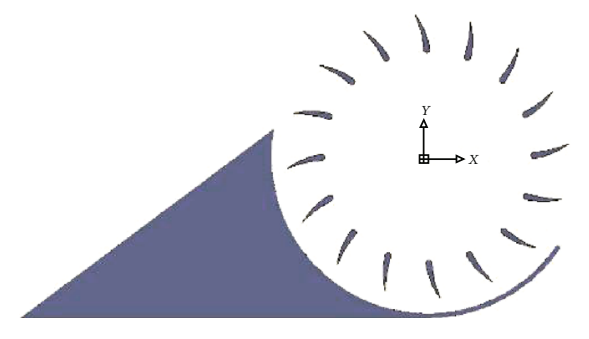

FIgURE 3: Fan-wing section with single leading edge winglet.

was positive. Figure 3 shows a whole Fan-wing with a single leading edge winglet.

2.3. Numerical Calculation Method. The maximum tip velocity of the crossflow fan was less than $0.3 \mathrm{Ma}$ (the maximum speed of the crossflow fan was defined as $2000 \mathrm{rpm}$ ), so the flow field in the numerical calculation can be assumed to be incompressible. Because of the cutting, rotating, and accelerating effects of crossflow fans on the flow field, the flow field was in an unsteady state. The Reynolds number should be considered, and the Reynolds number was defined as $5.927 \times 10^{5}$. The numerical simulation software used was FLUENT 14.5. N-S (Navier-Stokes) equation, RNG (Renormalization-group) turbulence model, SIMPLE (semi-implicit method for pressure-linked equations) pressure-velocity coupling algorithm and 2-order upwind convection term has been selected. The whole flow field was a finite body with mixed grids. The computational domain was shown in Figure 4(a). Nonslip boundary conditions were used on the airfoil wall. Structural grids are used in the annular area, the internal area, and the outer area of the dense area where the crossflow fan blades are located (Figure 4(b)). Nonstructural grids were used mainly in the outer area of the annulus and the elliptical dense area of the airfoil and near the blades of the crossflow fan. The grid and slip interface were shown in Figure 4(c) and the grid near the leading edge wing was shown in Figure 4(e). The whole flow field grid was shown in Figure 4(e). The length and width of the flow field was $14 \times 8 \mathrm{~m}$ and the number of grids was about 420,000. Mesh independency studies were also conducted with three successive meshes: coarse, medium, and fine meshes. The coarse mesh has 294,416 elements. The medium mesh has 419,156 elements. The fine mesh has 782,666 elements. The study has shown that differences in results from the coarse and medium meshes are significant, while differences in results between the medium and fine meshes are almost negligible. Hence, it was decided to use the medium mesh as the baseline for further simulation. For this simulation, a time step size equal to $1 / 20$ th the blade passing period captured the unsteady flow, within each time step, iterations were performed until the solution no longer changed. The result is considered to be convergent.

2.4. Examples Verification. In order to verify the accuracy of the numerical algorithm, the results were compared with those of the wind tunnel test of the National Key Laboratory of Rotorcraft Aeromechanics (Figures 5(a) and 5(c)Figure 5(c)). The length of the test model of Fan-wing was $500 \mathrm{~mm}$. The crossflow fan was made of carbon fibers. The airfoil was made of glass fiber. The lift coefficient and thrust coefficient of the Fan-wing model in the inflow velocity of $10 \mathrm{~m} / \mathrm{s}$, angle of attack of 0 degree and the cross-flow fan speed range of 400-1200 rpm have been calculated, respectively. From the Figures 5(d) and 5(e), it can be seen that the numerical results of Fan-wing were in good agreement with the experimental results. The maximum error was less than $10 \%$, and the error of the crossflow fan at high speed was less than 5\%. Therefore, the numerical algorithm adopted in this paper can be used to simulate and analyze the aerodynamic characteristics of the leading edge of the Fan-wing.

\section{Calculation Results and Analysis}

Using the numerical calculation method mentioned above, the angle of attack of the Fan-wing was calculated to be 0 , 4,8 , and 12 degrees, the inflow velocities were $8 \mathrm{~m} / \mathrm{s}$, $12 \mathrm{~m} / \mathrm{s}, 16 \mathrm{~m} / \mathrm{s}$, and $20 \mathrm{~m} / \mathrm{s}$, respectively, and the speed of the cross-flow fan was set to $2000 \mathrm{rpm}$. The positive angle of attack was chosen to ensure that the Fan-wing can always generate forward thrust.

\subsection{Aerodynamic Characteristics of Leading Edge Winglets with Different Numbers of Blades}

3.1.1. Single Leading Edge Winglet. The calculated inflow was $12 \mathrm{~m} / \mathrm{s}$, the angle of attack of the fan start at 0 degree, terminate at 12 degrees, and increase by 4 degrees, and the rotation angle of the leading edge winglet started at 0 degree, 


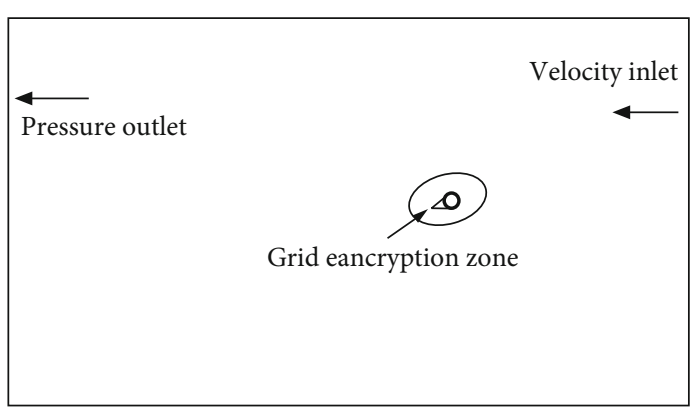

(a) Computational domain

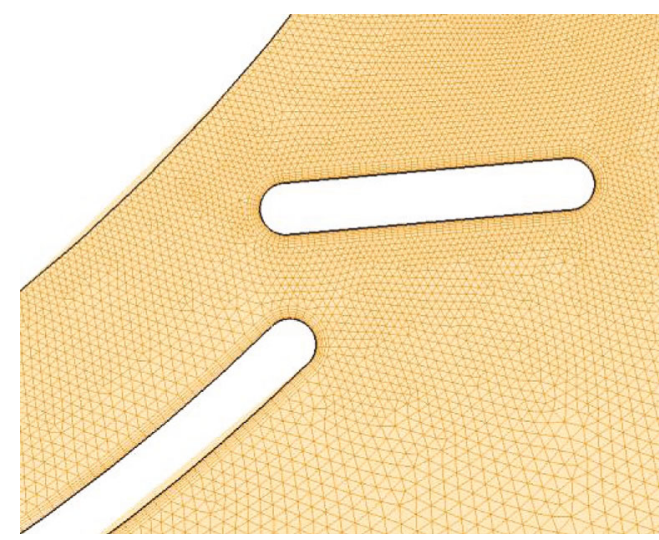

(d) Mesh at leading edge winglet

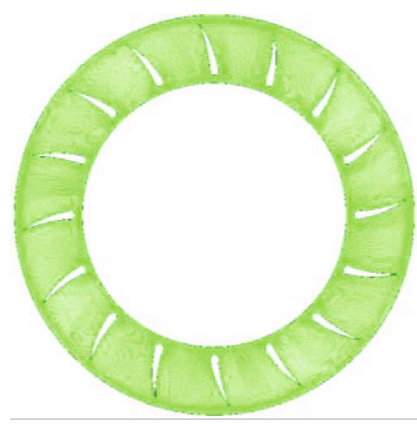

(b) Mesh generation in ring region

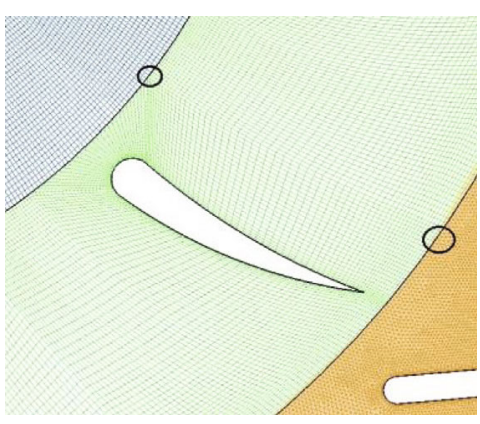

(c) Slip interface

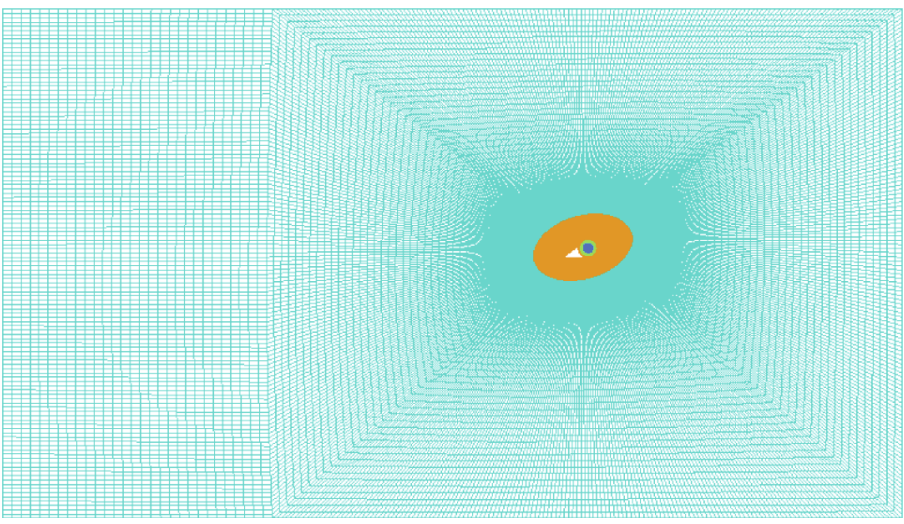

(e) All flow grid

FIgURE 4: Mesh generation.

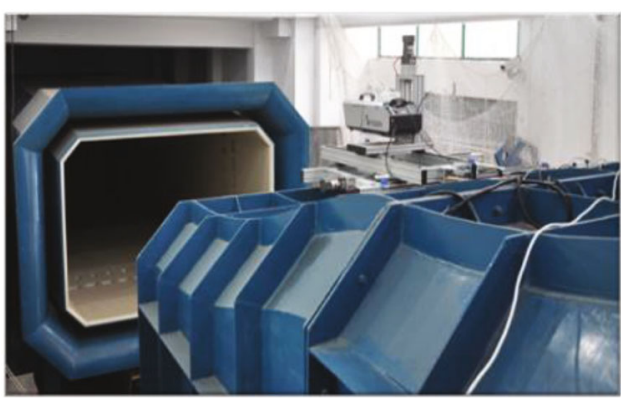

(a) Wind tunnel

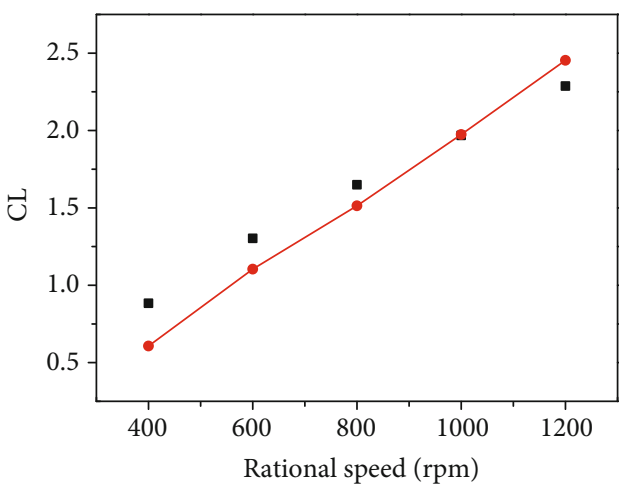

- EXP

$\rightarrow$ CFD

(d) Lift coefficient

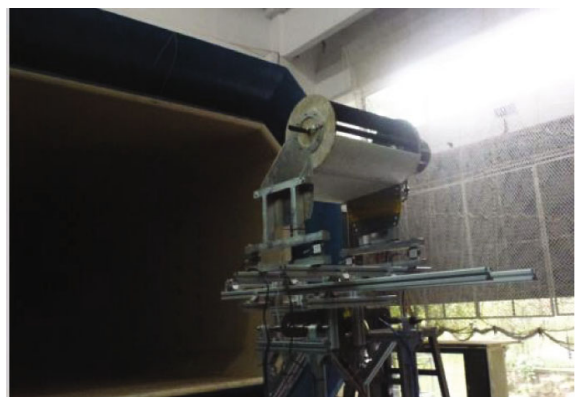

(b) Test bench

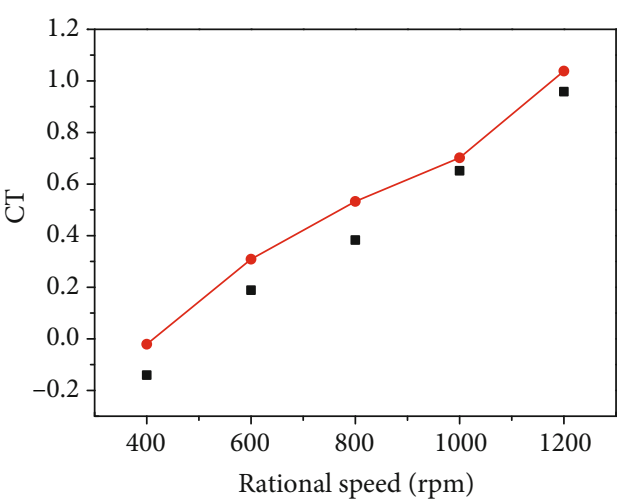

- EXP

$\rightarrow$ CFD

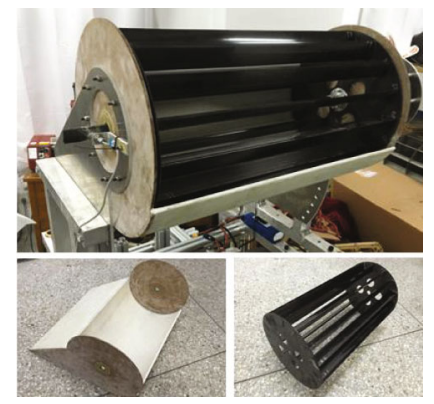

(c) Test model

(e) Thrust coefficient

FIGURE 5: Comparisons between numerical calculation and experimental results. 


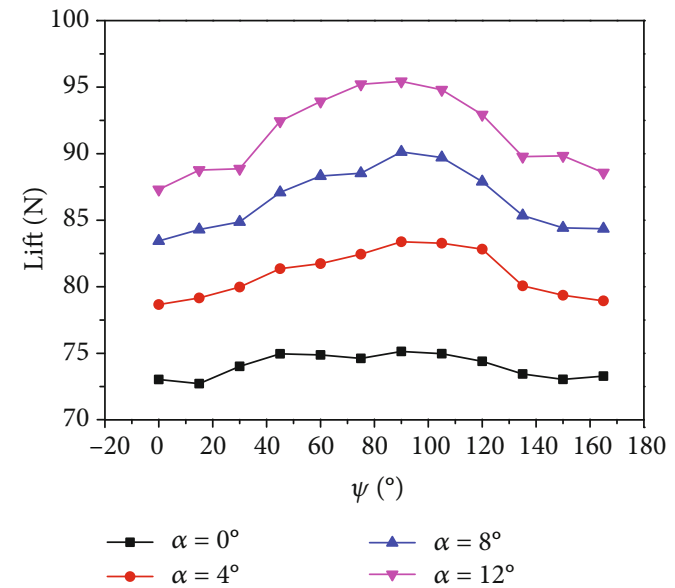

(a) Lift

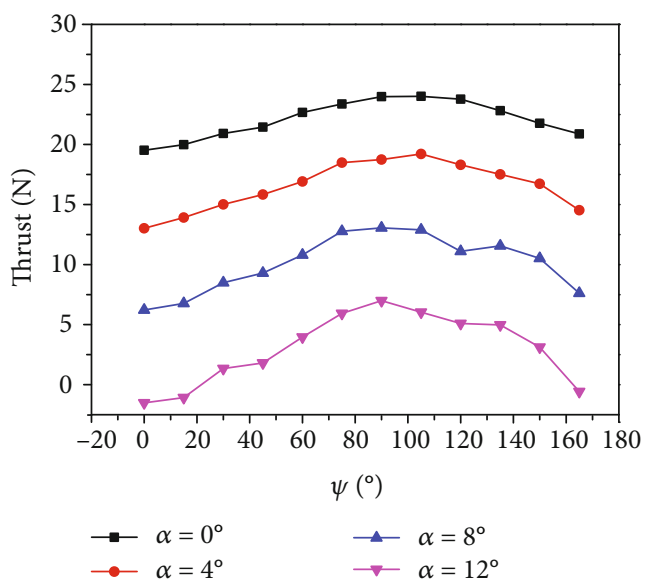

(b) Thrust

FIGURE 6: The variation curve of installation angle of a single leading edge winglet with angle of attack $(v=12 \mathrm{~m} / \mathrm{s})$.

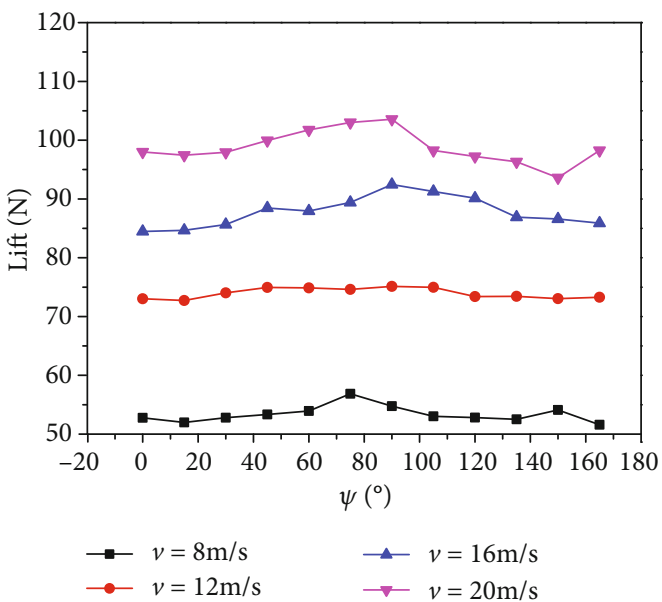

(a) Lift

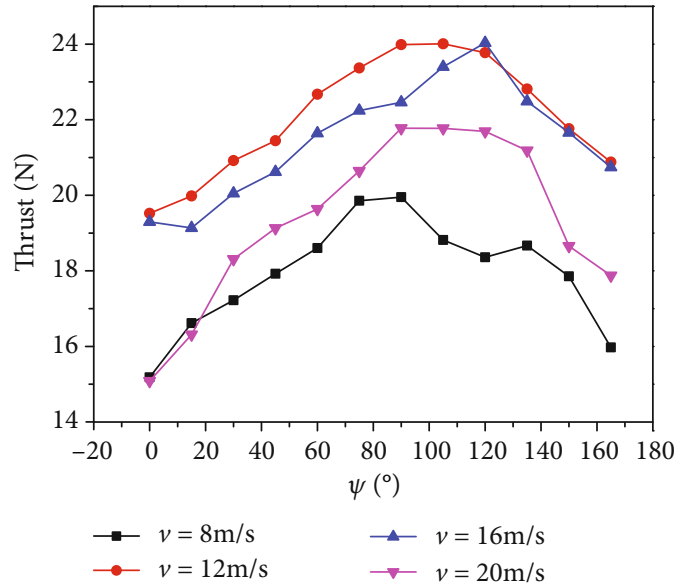

(b) Thrust

Figure 7: The variation curve of the installation angle of the single leading edge winglet with the flow $\left(\alpha=0^{\circ}\right)$.

terminate at 165 degrees, and increase by 15 degrees. As can be seen from Figure 6, when the angle of attack was unchanged, the lift and thrust increase first and then decrease with the increase of the deflection angle of the winglet. The lift and thrust of the winglet reach their peak value when the deflection angle of the winglet was near 90 degrees. With the increase of the angle of attack, the variation of the lift and thrust becomes larger. It can also be seen from the figure that when the deflection angle of the leading edge winglet remains unchanged, with the increase of the angle of attack of the Fan-wing, the lift force increases, while the thrust decreases gradually.

Figure 7 shows the variation of lift and thrust with the inflow velocity and the deflection angle of the leading edge winglet at an angle of attack of 0 degree. It can be seen from the figure that when the inflow velocity was constant, with the increase of the deflection angle of the leading edge wing, the lift and thrust increase first and then decrease, and the lift change was small, and the thrust change was large. When the inflow velocity was $12 \mathrm{~m} / \mathrm{s}$ and the angle of attack was 0 degree, the thrust increases by $22.97 \%$ with the increase of the deflection angle, while the lift increases by only $6 \%$. This shows that the increase of the leading edge winglet has an obvious effect on the lift of the Fan-wing. In the figure, the thrust reaches its maximum at the leading edge winglet deflection angle from 90 degrees to 120 degrees. When the angle of attack and the deflection angle of the leading edge wing remain unchanged, the larger the inflow velocity was, the larger the lift of the Fan-wing was.

The velocity nephogram of a single leading edge winglet at different deflection angles has been further analyzed (Figure 8). As can be seen from the figure, during the rotation process of a single leading edge winglet (i.e., the increase of the deflection angle of the leading edge winglet), the opening angle of the leading edge of the fan actually increases from 25 degrees to 35 degrees. When the deflection angle of the 


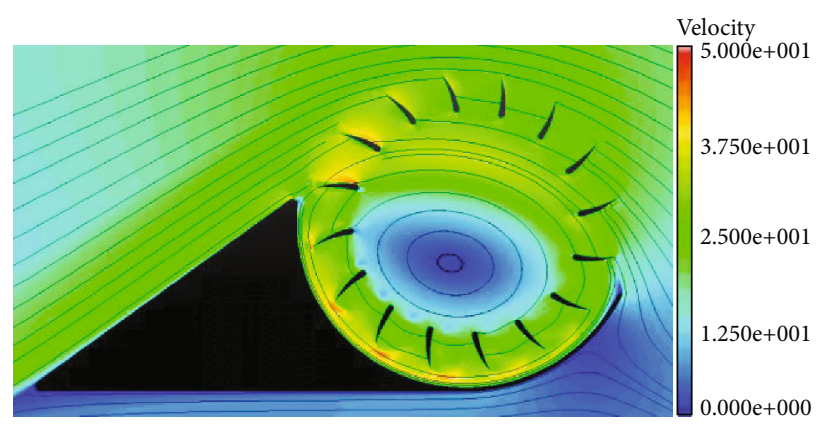

(a) Deflection angle $=0^{\circ}$

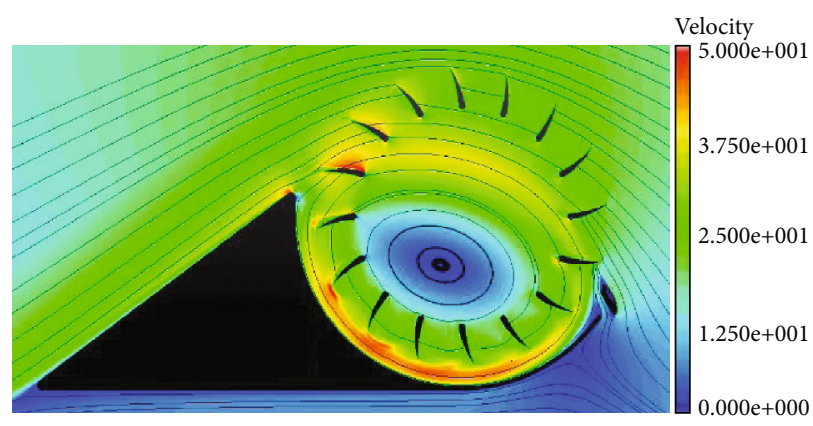

(c) Deflection angle $=60^{\circ}$

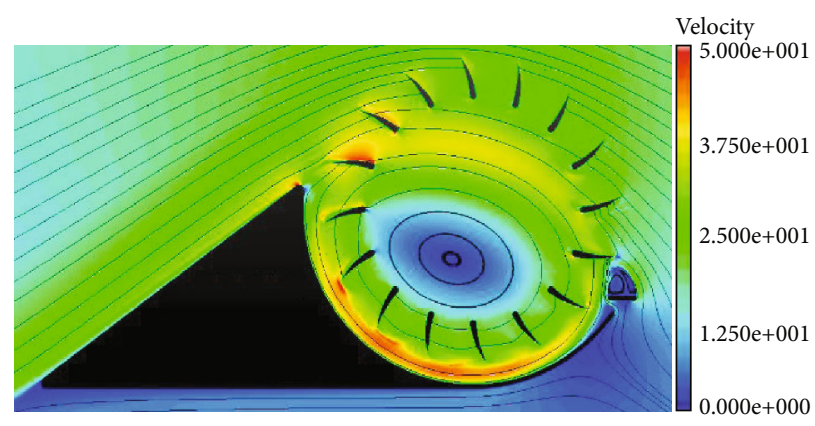

(e) Deflection angle $=120^{\circ}$

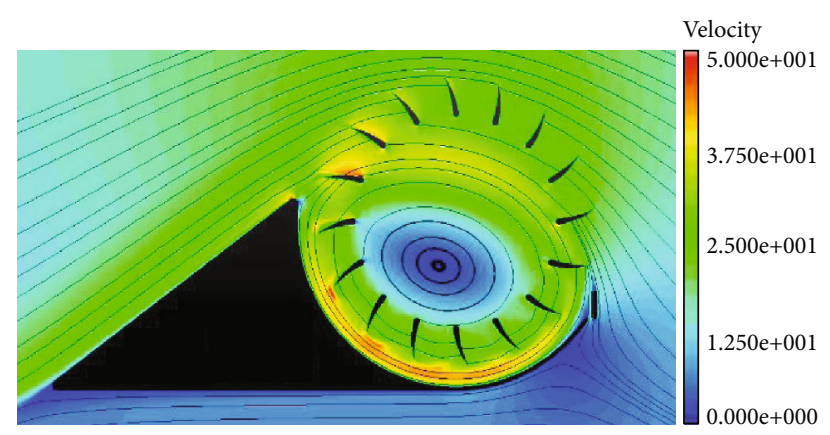

(b) Deflection angle $=30^{\circ}$

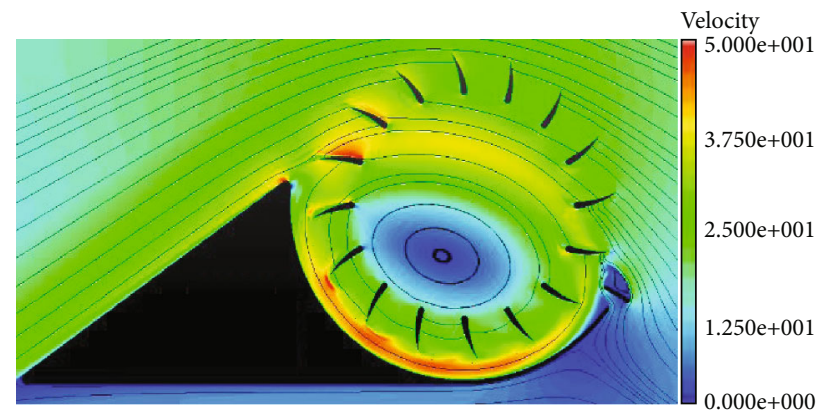

(d) Deflection angle $=90^{\circ}$

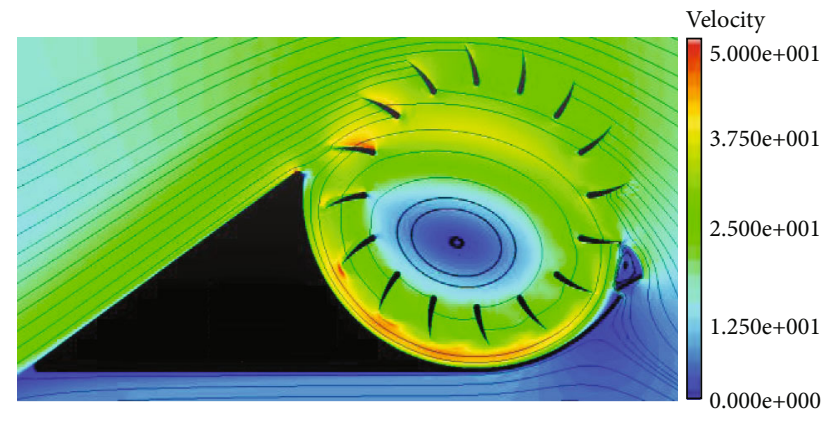

(f) Deflection angle $=150^{\circ}$

FIGURE 8: Velocity nephogram and streamline diagram of a single leading edge winglet $\left(\nu=12 \mathrm{~m} / \mathrm{s}, \alpha=0^{\circ}\right)$.

winglet changes, the inflow will flow into the crossflow fan from the gap between the leading edge of the winglet and the leading edge of the arc groove, and then be accelerated by the crossflow fan, thus changing the direction of the inflow. When the deflection angle of the small wing was small, the inflow direction was close to the chord direction of the winglet. At this time, the deflection angle of the leading edge of the small wing was small, and the lift and thrust increase slowly. When the deflection angle of the winglet reaches 90 degrees, the inflow into the crossflow fan was the most. At this time, the resistance of the winglet to the inflow was small, and the lift and thrust of the fan reach the maximum. When the deflection angle of the winglet continues to increase ( $>90$ degrees), the inflow into the crossflow fan gradually decreases, and the inflow was perpendicular to the chord direction of the leading edge wing. The inflow was blocked by the leading edge winglet, and wake vortices were generated in the rear part. Although the winglet can also generate some lift at this time, the existence of the wake was equivalent to reducing the leading edge opening angle, reduc- ing the inflow flow into the crossflow fan, which reduces the lift and thrust of the Fan-wing.

The pressure nephograms of a single leading edge winglet at different deflection angles were analyzed and compared (Figure 9). With the increase of the deflection angle of the leading edge winglet, the pressure in the elliptical lowpressure vortex region of the crossflow fan decreases first and then increases. The pressure of the low-pressure vortex was the lowest near the deflection angle of the leading edge winglet at 90 degrees. The change of the deflection angle of the leading edge winglet increases the surface pressure on the arc groove of the leading edge of the Fan-wing.

Combining with the pressure distribution curve of the upper and lower surfaces of the Fan-wing airfoil (Figure 10), the effect of a single leading edge winglet on the aerodynamics of the Fan-wing can be seen more intuitively. From the figure, it can be seen that the deflection angles were affected by the low-pressure vortices inside the crossflow fan. The incoming flow flows into the crossflow fan from the gap between the winglet and the arc groove, 


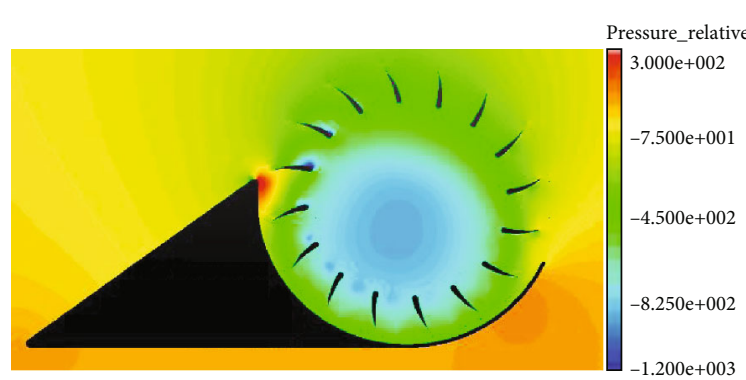

(a) Deflection angle $=0$ 。

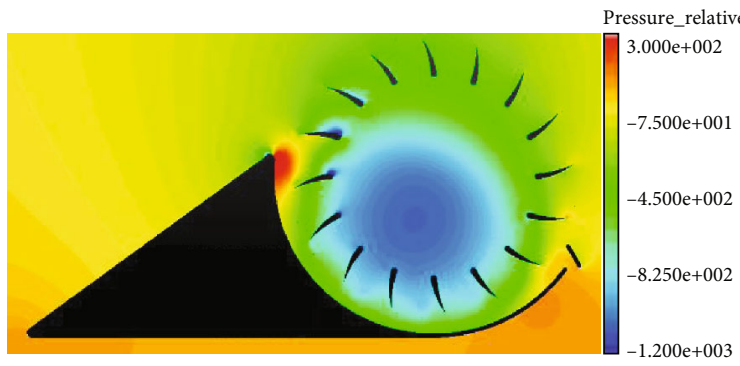

(c) Deflection angle $=60^{\circ}$

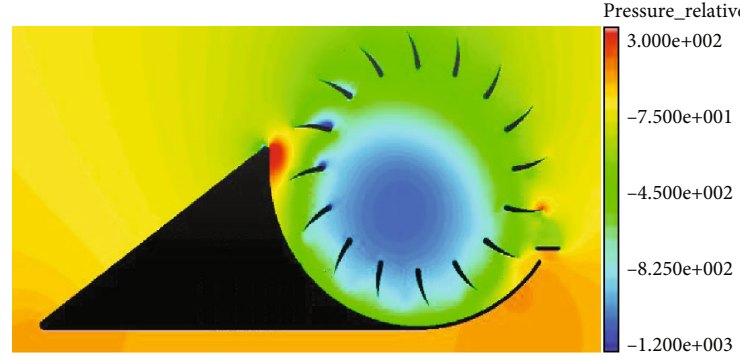

(e) Deflection angle $=120^{\circ}$

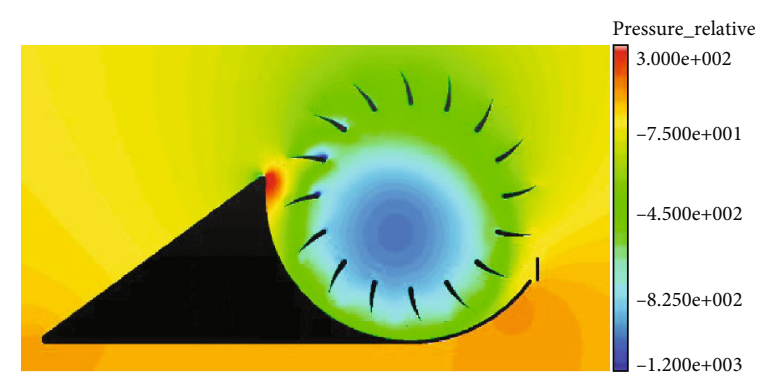

(b) Deflection angle $=30^{\circ}$

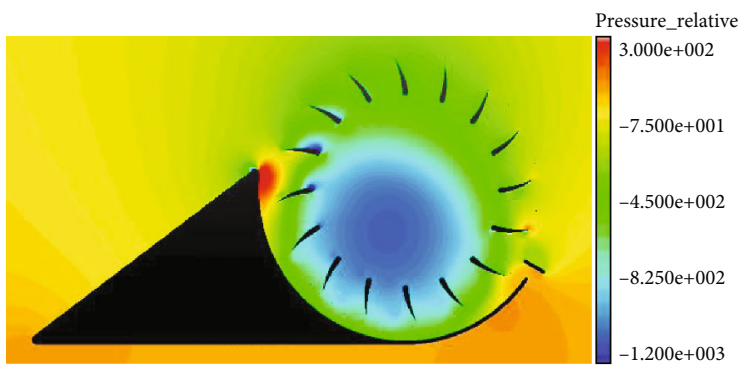

(d) Deflection angle $=90^{\circ}$

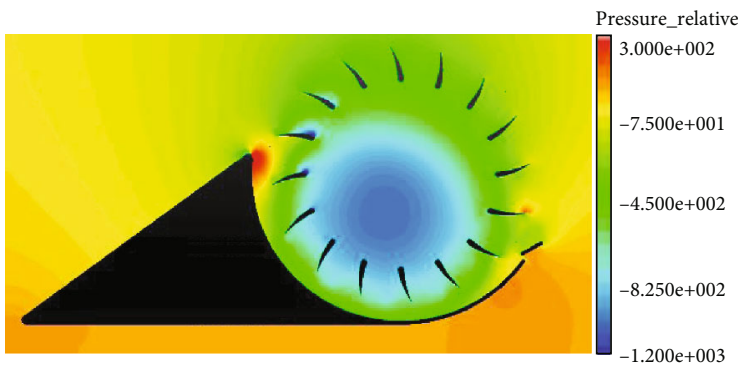

(f) Deflection angle $=150^{\circ}$

Figure 9: Pressure nephogram of a single leading edge winglet $\left(v=12 \mathrm{~m} / \mathrm{s}, \alpha=0^{\circ}\right)$.

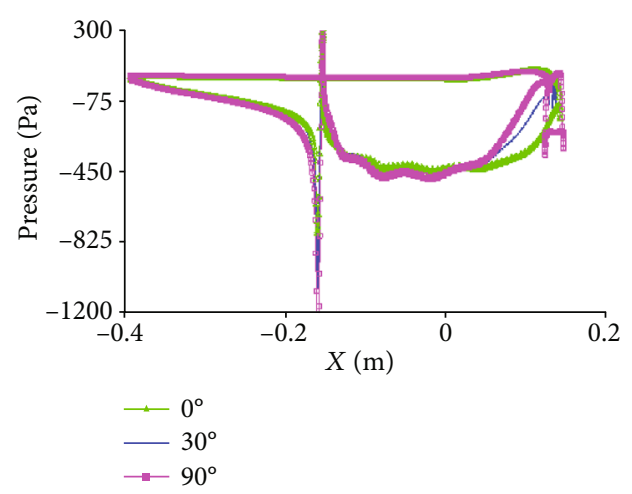

Figure 10: Static pressure distribution curve on the surface of the Fan-wing with single leading edge winglet $\left(v=12 \mathrm{~m} / \mathrm{s}, \alpha=0^{\circ}\right)$.

which increases the pressure near the leading edge of the arc groove. The larger the deflection angle of the winglet, the more the pressure increases. When the deflection angle of the current edge wing was near 90 degrees, the relative pressure difference between the inner and outer surfaces of the winglet was large, which can provide some lift. On the whole, however, the effect of different deflection angles of a single leading edge winglet on the total lift of the fan was relatively small.

3.1.2. Double Leading Edge Winglets. Figure 11 shows the lift and thrust curves of double leading edge winglets at different deflection angles. The deflection angle of double leading edge winglets increases and decreases simultaneously. With the increase of the winglet deflection angle, the thrust and lift increase first and then decrease and reach the maximum when the deflection angle was near 90 degrees. When the angle of attack was 0 degrees, the thrust increases by about $25 \%$, while the lift increases by only about $4 \%$.

Figure 12 shows the curves of the deflection angles of double leading edge winglets varying with the inflow when the angle of attack of the Fan-wing was 0 degrees. It can be seen from the figure that with the increase of the deflection angle of the double leading edge winglets, the thrust increases first and then decreases, and the maximum value was obtained near 90 to 120 degrees while the lift varies little with the deflection angle. When the deflection angle was greater than 120 degrees, the lift decreases. In addition, it can be seen from the figure that with the increase of the inflow velocity, 


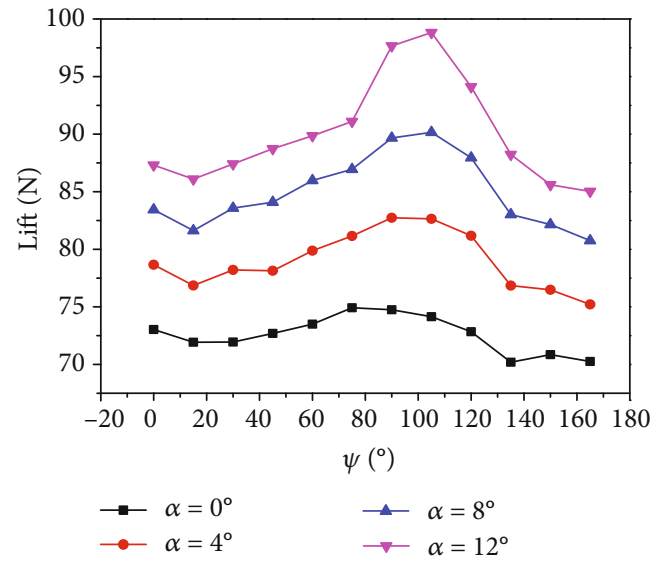

(a) Lift

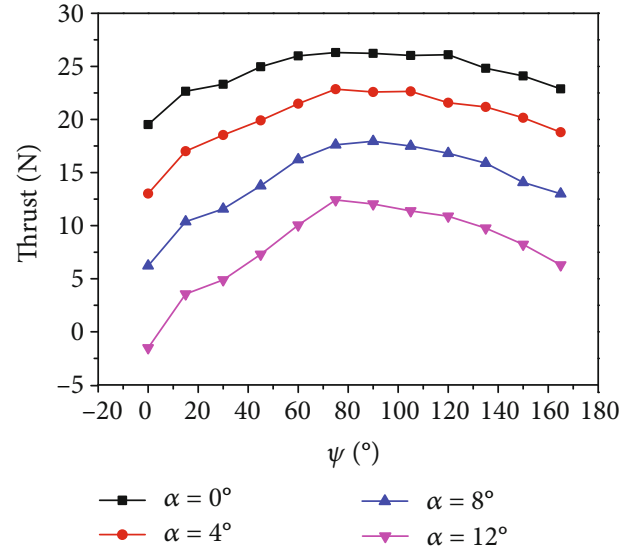

(b) Thrust

FIGURE 11: The variation curve of installation angle of a double leading edge winglet with angle of attack $(v=12 \mathrm{~m} / \mathrm{s})$.

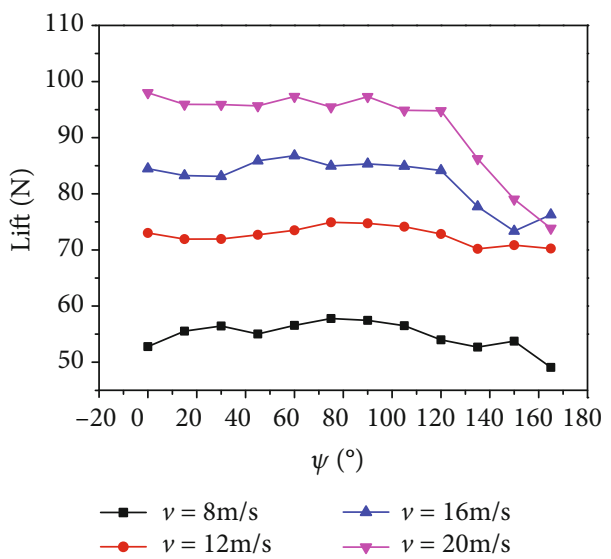

(a) Lift

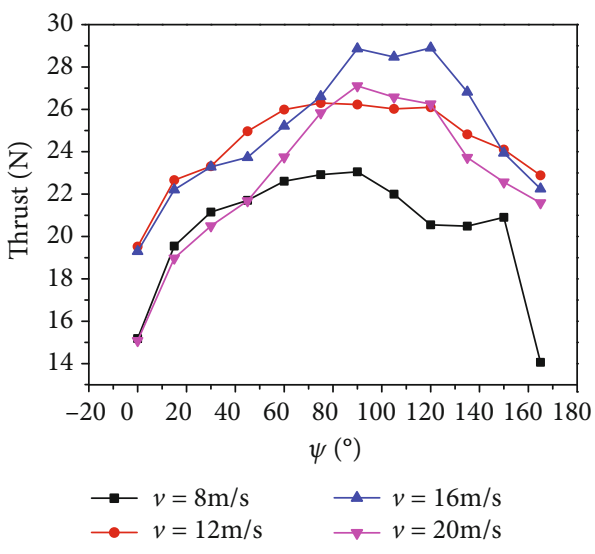

(b) Thrust

FIgURE 12: The variation curve of the installation angle of the double leading edge winglet with the flow $\left(\alpha=0^{\circ}\right)$.

the thrust increases first and then decreases, while the lift increases gradually, but the increase decreases gradually.

The velocity nephogram of double leading edge winglets at different inflow velocities was further analyzed (Figure 13). As can be seen from the figure, with the increase of the inflow velocity, the change of the inflow direction of the crossflow fan decreases, and the inflow flow into the crossflow fan increases, which increases the flow velocity on the inner surface of the arc groove and the inclined surface of the trailing edge of the fan, and the low-pressure eccentric vortices are squeezed to move downward and left. The small increase of lift was mainly due to the increase of deflection angle of leading edge winglet, the lift surface of the arc groove section of the leading edge was partially destroyed, resulting in the loss of lift, and the increase of the opening angle of the Fan-wing makes the inflow into the crossflow fan increase, resulting in the increase of total lift. When the deflection angle of the leading edge wing changes, the influence of the two factors on the lift of the wing was small. Generally speaking, with the increase of the angle of attack, the relative increment of lift and thrust increases gradually. At the same time, the maximum thrust corresponds to the decrease of the deflection angle of the leading edge winglet, while the maximum lift corresponds to the increase of the deflection angle of the leading edge winglet.

From the static pressure distribution curve of the upper and lower surfaces of the Fan-wing in Figure 14, it can be seen that with the increase of the inflow velocity, the pressure on the inner surface of the oblique and arc grooves of the rear edge of the fan decreases, the pressure on the lower surface of the airfoil increases slightly, and the pressure at the stagnation point of the leading edge increases gradually, and the pressure difference at the winglet decreases due to the dispersal of the wake vortices.

3.1.3. Triple Leading Edge Winglets. The triple leading edge winglets make the opening angle of the leading edge of the Fan-wing increase from 25 to 55 degrees. Figure 15 shows the variation of lift and thrust with the deflection angle and attack angle of triple leading edge winglets when the inflow 


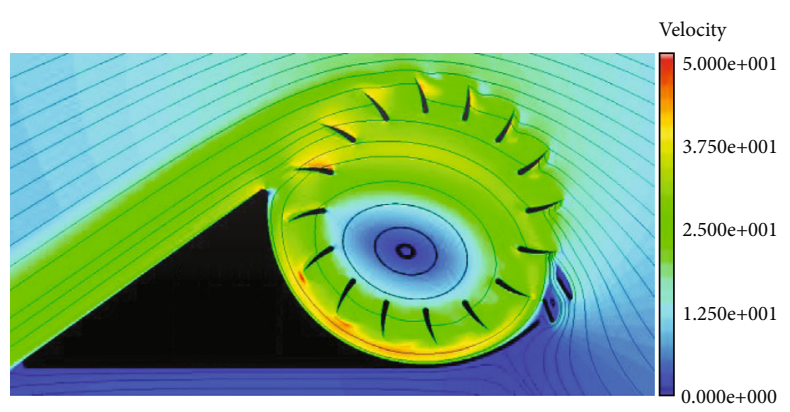

(a) Inflow velocity $=8 \mathrm{~m} / \mathrm{s}$

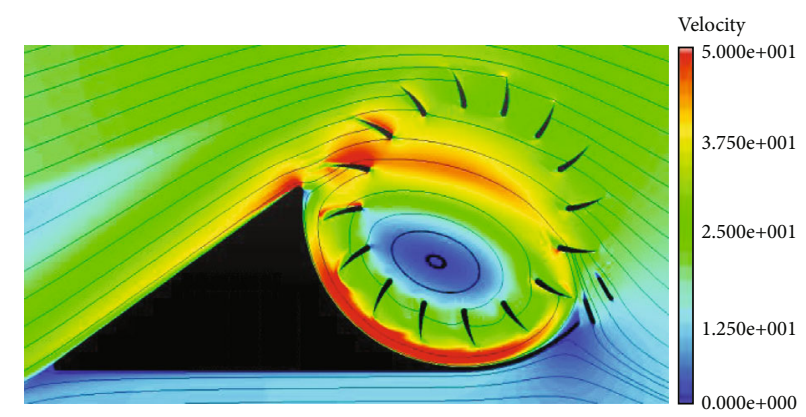

(c) Inflow velocity $=16 \mathrm{~m} / \mathrm{s}$

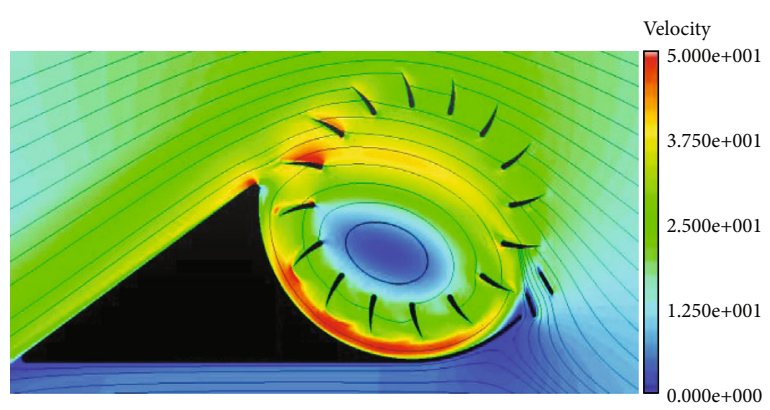

(b) Inflow velocity $=12 \mathrm{~m} / \mathrm{s}$

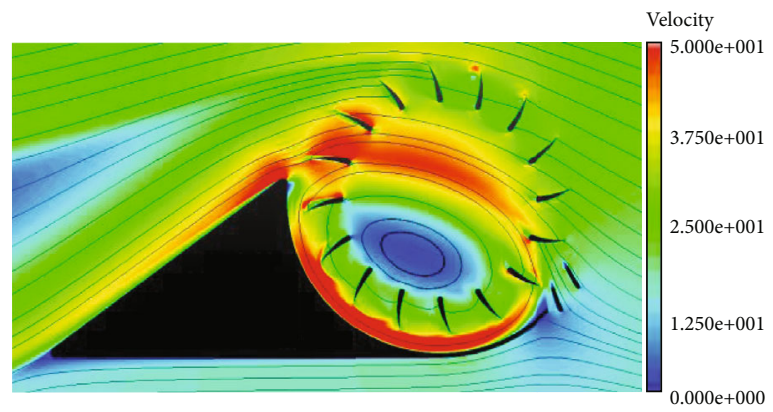

(d) Inflow velocity $=20 \mathrm{~m} / \mathrm{s}$

Figure 13: Velocity nephogram and streamline diagram of a double leading edge winglet (installation angle $=60^{\circ}, \alpha=0^{\circ}$ ).

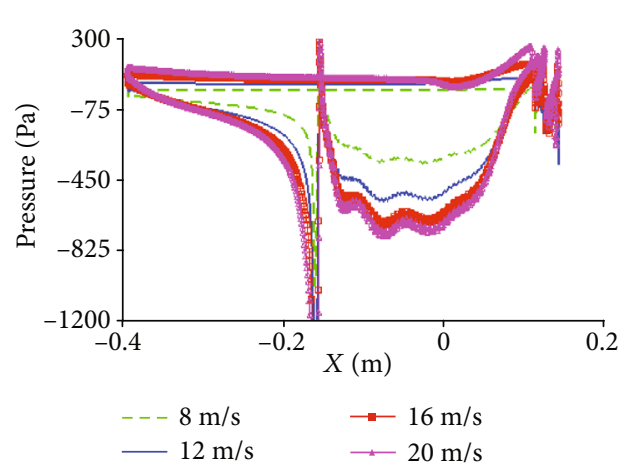

FIGURE 14: Static pressure distribution curve on the surface of the Fan-wing with a double leading edge winglet (installation angle $=60^{\circ}$, $\alpha=0^{\circ}$ ).

was constant. It can be seen from the figure that with the increase of the deflection angle, the lift of the wing decreases first, then increases and then decreases, and the maximum value was obtained near the deflection angle of the winglet from 75 to 105 degrees; the thrust of the fan first increases and then decreases, and the maximum value was obtained near the deflection angle of the winglet from 90 to 120 degrees.

Figure 16 shows the lift and thrust curves of a Fan-wing with triple leading edge winglets fixed at the same angle of attack with respect to the deflection angle and inflow of the winglet. It can be seen from the figure that with the increase of the deflection angle of the leading edge winglet, the lift increases first and then decreases when the inflow velocity was small, and the maximum value was obtained near the deflection angle of the 90 degree winglet; the thrust first increases and then decreases, and the maximum value was obtained near the deflection angle of the 75 degree to 120 degree winglet. The thrust does not change much with the increase of the incoming velocity, but decreases with the increase of the current velocity.

The velocity nephograms of triple leading edge winglets with different deflection angles were further analyzed (Figure 17). It can be seen from the figure that with the increase of the deflection angle of the triple leading edge wings, the flow velocity of the crossflow fan to the inflow direction, the leading edge arc groove and the trailing edge slope of the Fan-wing show the trend of first increasing and then decreasing. When the deflection angle of triple leading edge winglets was small or large, the change of the opening angle of the leading edge of the fan is small, which makes the inflow flow into the crossflow fan change little. However, the existence of the deflection angle of the small wing destroys the stability of the original arc-groove lift surface and causes the interior of the crossflow fan. Variations in the position and intensity of eccentric vortices cause fluctuations in lift and thrust of the fan. When the deflection angle of triple leading edge winglets was between 90 and 105 degrees the opening angle of the winglet was the largest, and more incoming flow enters the crossflow fan, which makes the lift and thrust of the winglet higher. When the inflow velocity continues to increase, it was not conducive to the formation of eccentric vortices in cross-flow fans. At the same time, the rotation of the leading edge winglet makes the size of the arc groove section of the leading edge of the fan smaller, resulting in the reduction of the airfoil area, which makes the lift and thrust of the fan decrease considerably at a larger deflection angle of the leading edge winglet, 


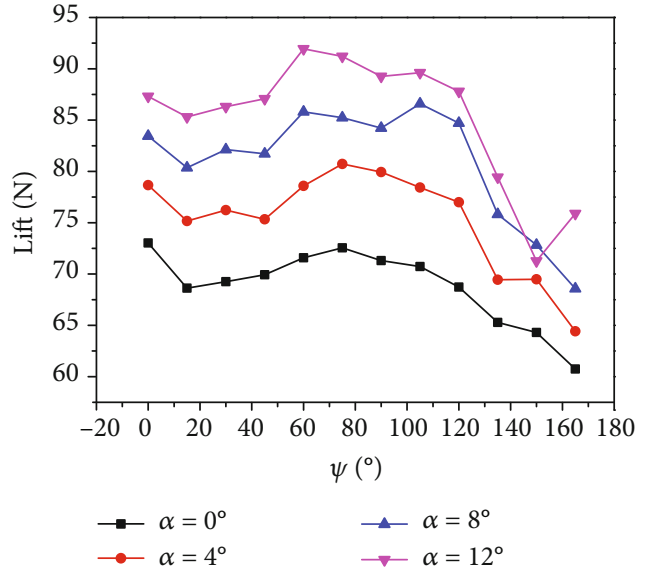

(a) Lift

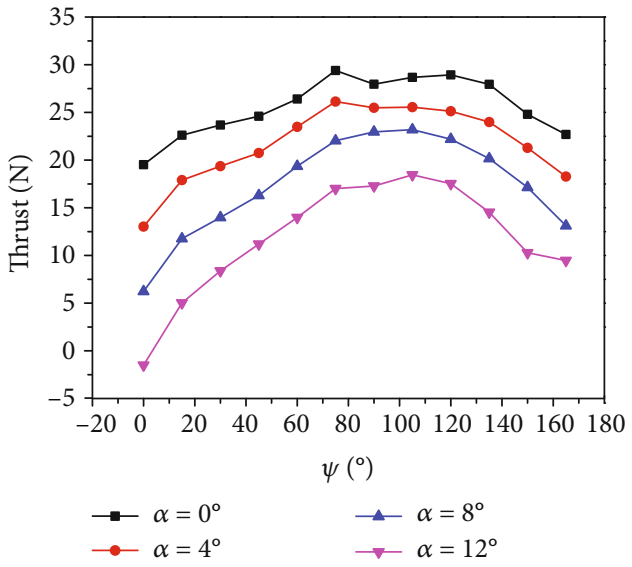

(b) Thrust

FIGURE 15: The variation curve of installation angle of a triple leading edge winglet with angle of attack $(v=12 \mathrm{~m} / \mathrm{s})$.

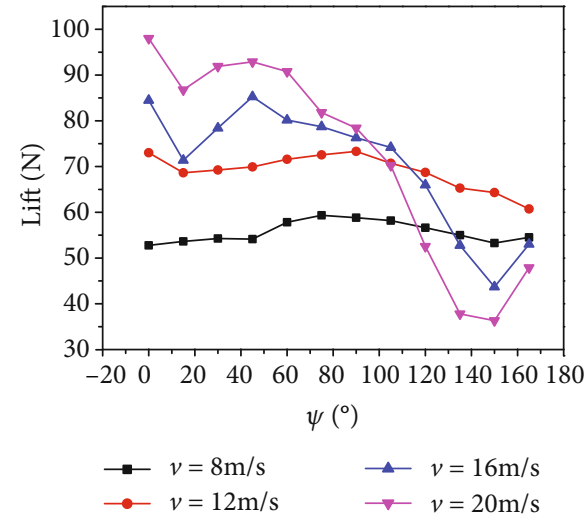

(a) Lift

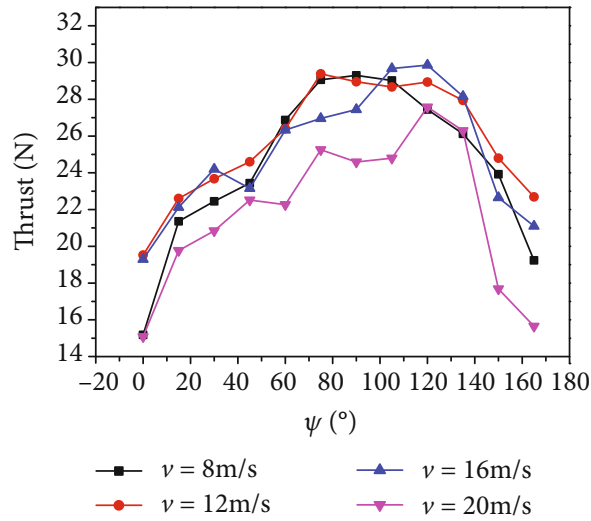

(b) Thrust

FIGURE 16: The variation curve of the installation angle of the triple leading edge winglet with the flow $\left(\alpha=0^{\circ}\right)$.

and the lift drop is particularly obvious. It can also be seen from the figure that triple leading edge winglets have a great change in the opening angle of the fan. When the deflection angle is greater than 90 degrees, wake vortices are formed near the winglet due to the blocking of the incoming flow. This will prevent the incoming flow from entering the cross-flow fan, which will have a negative impact on the lift and thrust of the Fan-wing.

The pressure nephograms at different deflection angles of leading edge winglets were analyzed and compared (Figure 18). With the increase of the deflection angle of triple leading edge winglets, the pressure of eccentric vortices in cross-flow fan decreases first and then increases. At the same time, when the deflection angle of triple leading edge winglets was small, the pressure difference of the appendages of the winglets in the horizontal direction decreases and was thrust. When the deflection angle of triple leading edge winglets was large, the winglets are nearly parallel to the chord of the winglets, and then small. The wing generates lift similar to the leading edge flaps.
From the static pressure distribution curve of the upper and lower surfaces of the fan in Figure 19(d), it can be seen that with the increase of the deflection angle of the triple small wings, the pressure at the front of the arc groove of the front edge of the fan increases greatly, while the pressure at the bottom of the arc groove of the front edge and the slope of the rear edge decreases. The sudden change of pressure in the installation area of the leading edge winglet can also reflect its influence on the lift thrust of the Fan-wing.

3.2. Comparisons of Aerodynamic Characteristics of Leading Edge Winglets with Different Numbers of Blades. Figure 20 is a velocity cloud and streamline diagram of different leading edge small fins with 90 degrees deflection angle, $12 \mathrm{~m} / \mathrm{s}$ inflow velocity, and 0 degrees attack angle. It can be seen from the figure that more incoming flow can enter the cross-flow fan when the leading edge winglet rotates, which actually increases the leading edge opening angle of the fan. With the increase of the number of leading edge winglets, the change of the leading edge opening angle of the fan 


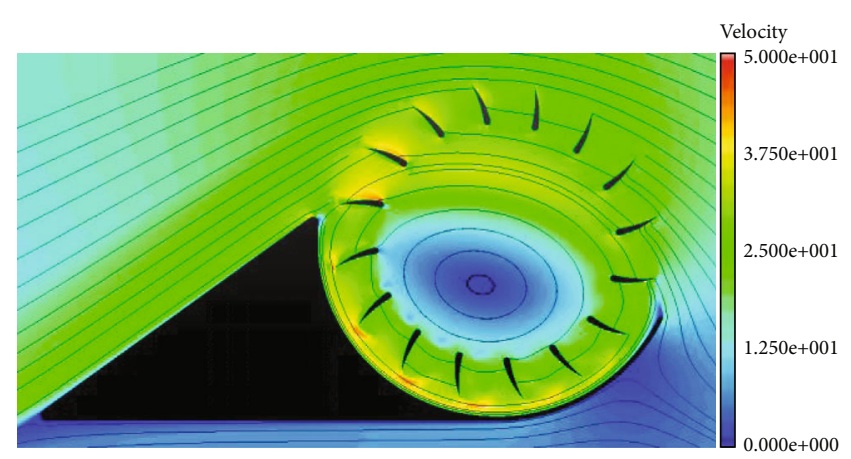

(a) Deflection angle $=0^{\circ}$

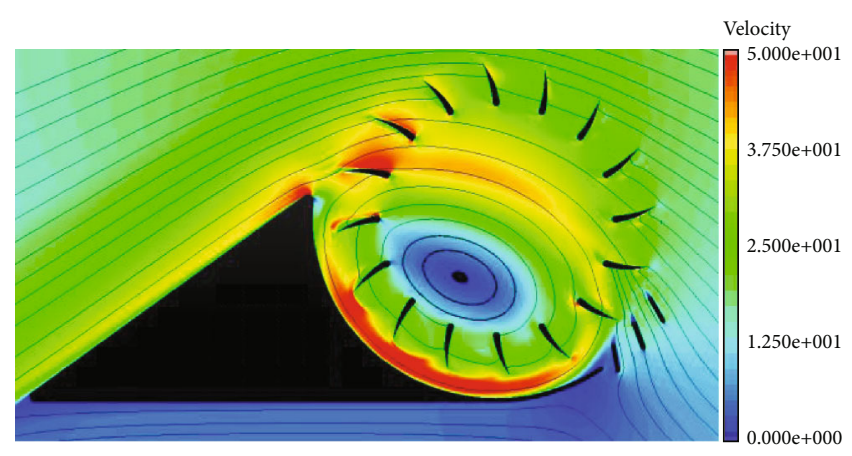

(c) Deflection angle $=60^{\circ}$

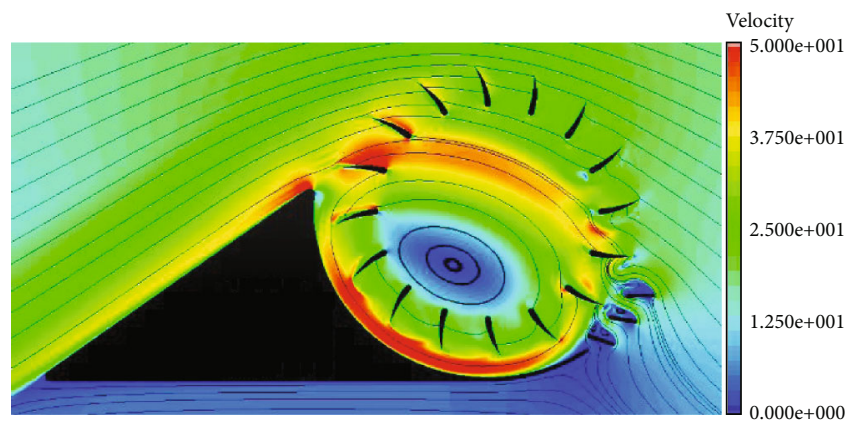

(e) Deflection angle $=120^{\circ}$

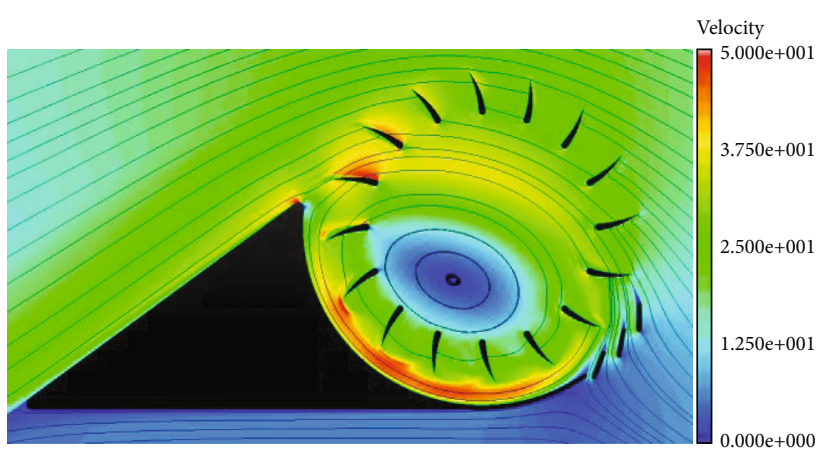

(b) Deflection angle $=30^{\circ}$

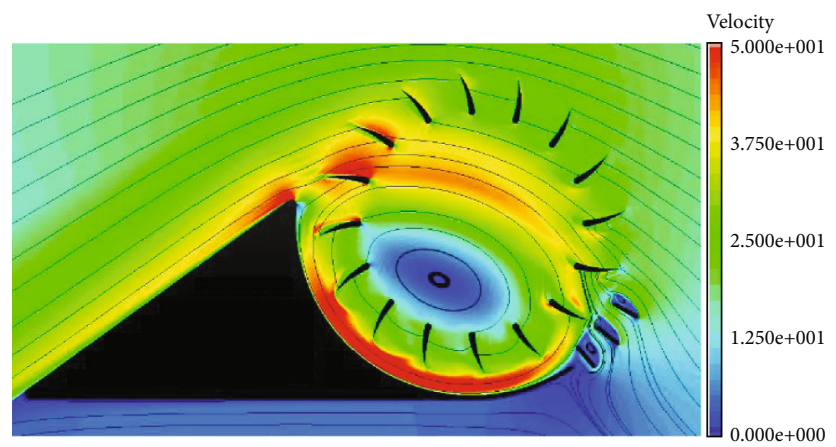

(d) Deflection angle $=90^{\circ}$

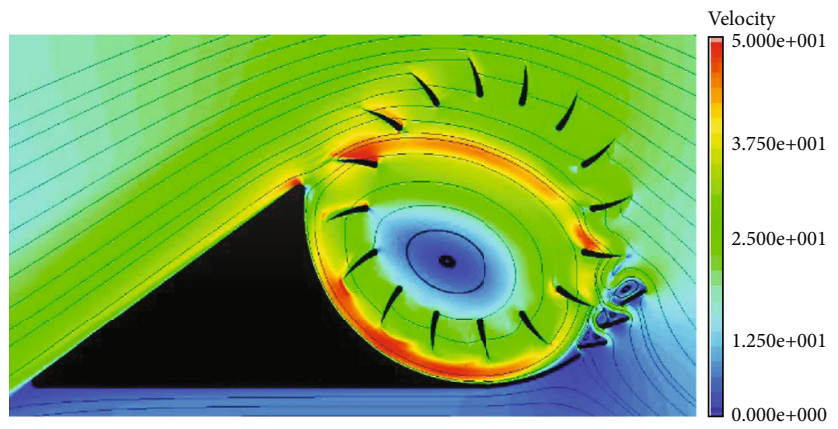

(f) Deflection angle $=150^{\circ}$

FIGURE 17: Velocity nephogram and streamline diagram of a single leading edge winglet $\left(v=12 \mathrm{~m} / \mathrm{s}, \alpha=0^{\circ}\right)$.

becomes larger, which makes the airflow velocity on the upper surface of the rear edge inclined plane and the leading edge arc groove increase gradually. The strength of lowpressure eccentric vortices in crossflow fans is further enhanced. The increase of the number of leading edge small fins also has an effect on the flow field near the small fins. The specific purpose is to increase the inflow angle of the incoming flow into the cross-flow Fan and the flow rate of the suction cross-flow fan.

According to the pressure nephogram of different leading edge small fins in Figure 21, with the increase of leading edge small fins, the intensity of low-pressure vortices in crossflow fans increases, the area increases, and the vorticity center moves to the left and rear. The influence of the vortex center position of crossflow fan can control the longitudinal moment of the fan, so that the leading edge wing can be used not only to improve the lift and thrust of the fan but also to control the attitude of the aircraft with the fan.
Comparing the static pressure distribution curves of the different number of small wings with the reference model in Figure 22, it can be found that the increase of the number of small wings reduces the pressure on the surface of the bevel of the rear edge and the arc groove of the front edge of the fan, and the more the number of small wings, the more obvious the pressure drop is. The pressure change of the lower wing surface is very small. Because of the rotation of the wing, there is a gap between the wing and the leading edge arc groove. The incoming flow can enter the crossflow fan through the gap, which makes the lift surface of the leading edge arc groove smaller and the pressure at the gap increase. Analysis of Figure 22(c) shows that the first leading edge winglet can provide larger lift, while the second and third leading edge winglets provide smaller lift. Figure 23 shows the relationship between lift and thrust with the different number of leading edge winglets. With the increase of the number of small leading edge winglets, the trend of lift and 


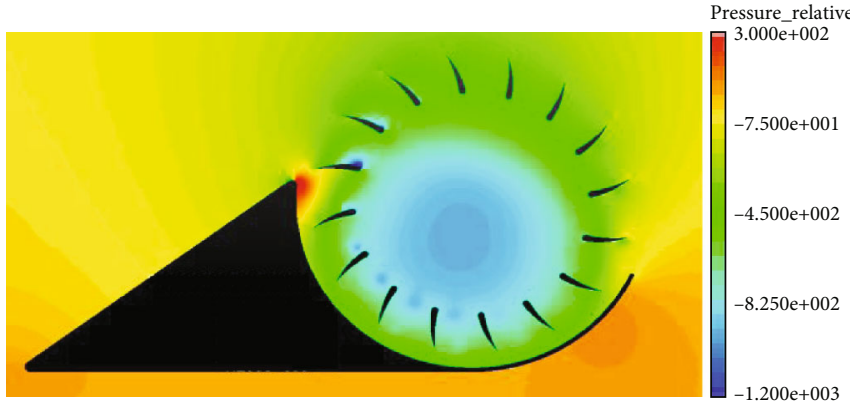

(a) Deflection angle $=0^{\circ}$

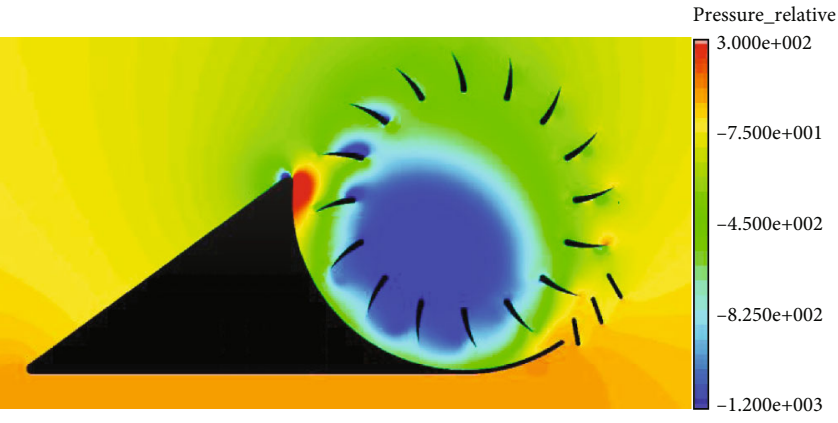

(c) Deflection angle $=60^{\circ}$

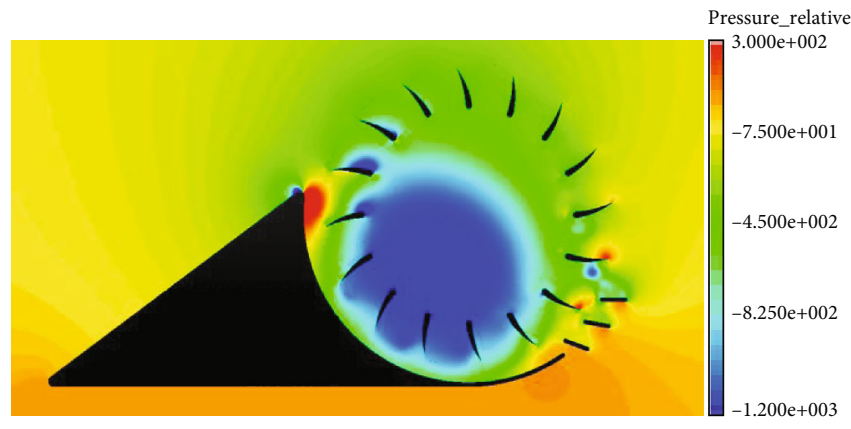

(e) Deflection angle $=120^{\circ}$

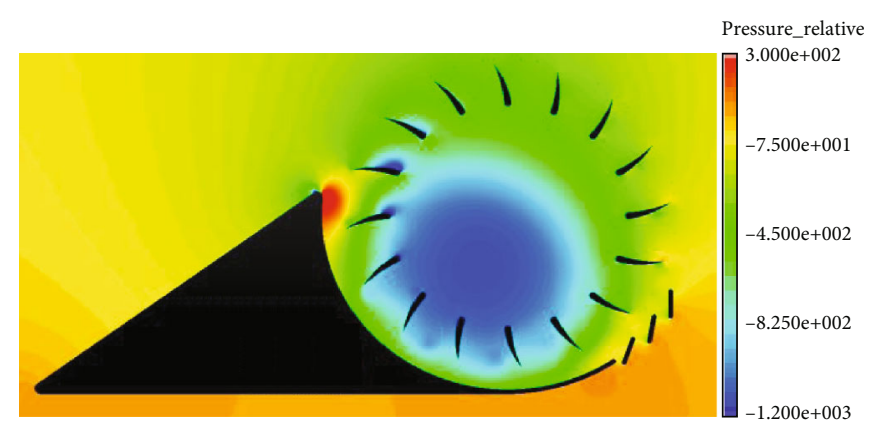

(b) Deflection angle $=30^{\circ}$

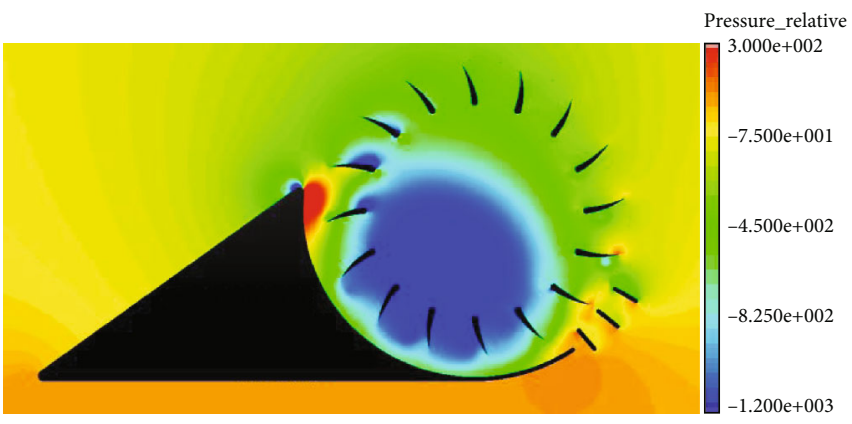

(d) Deflection angle $=90^{\circ}$

Pressure_relative

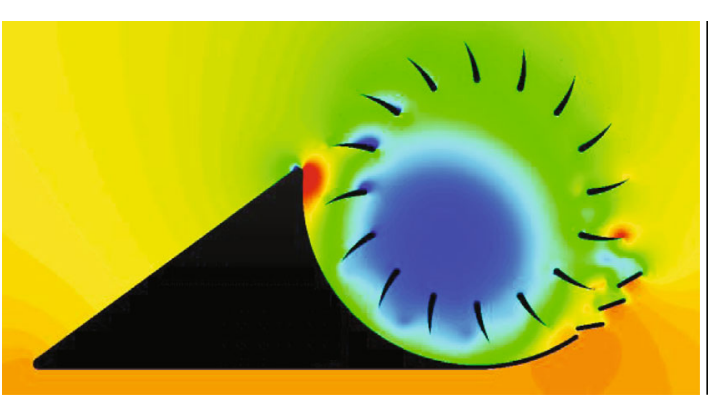

$3.000 \mathrm{e}+002$

$-7.500 \mathrm{e}+001$

$-4.500 \mathrm{e}+002$

(f) Deflection angle $=150^{\circ}$

FIGURE 18: Pressure nephogram of triple leading edge winglet $\left(v=12 \mathrm{~m} / \mathrm{s}, \alpha=0^{\circ}\right)$.

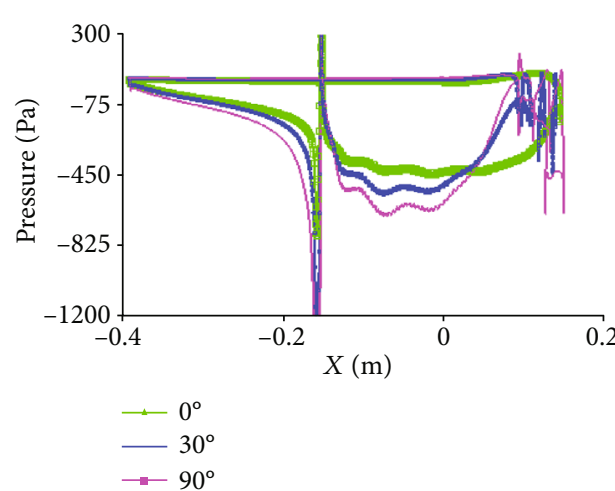

(a) Winglet deflection angle $0^{\circ}, 30^{\circ}$, and $90^{\circ}$

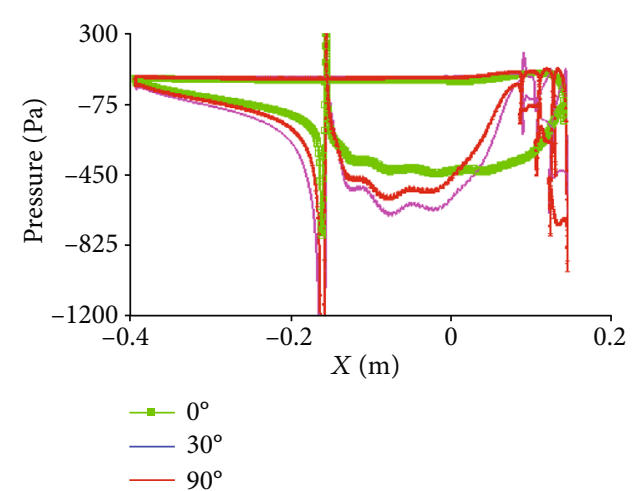

(b) Winglet deflection angle $0^{\circ}, 90^{\circ}, 150^{\circ}$

Figure 19: Static pressure distribution curve on the surface of the Fan-wing with single leading edge winglet $\left(v=12 \mathrm{~m} / \mathrm{s}, \alpha=0^{\circ}\right)$. 


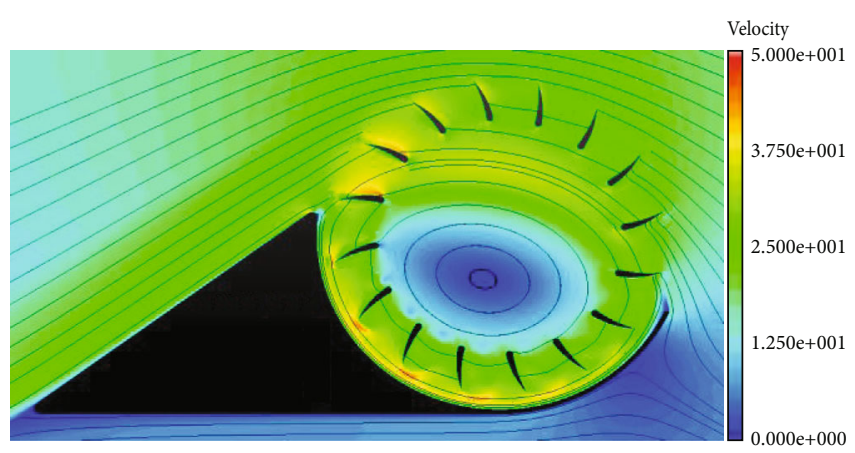

(a) No winglet

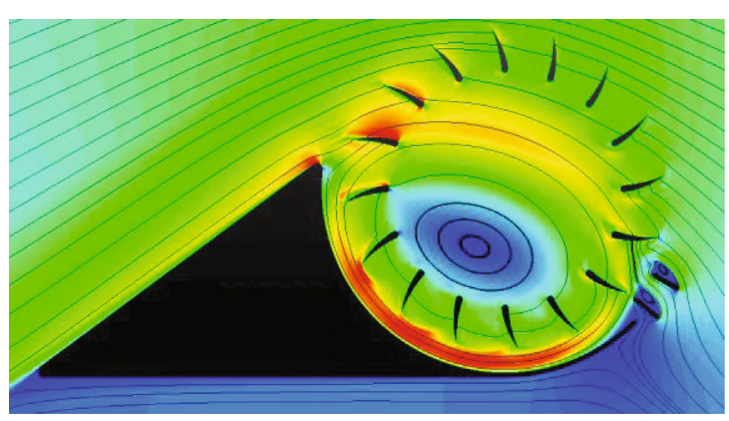

(c) Double winglet

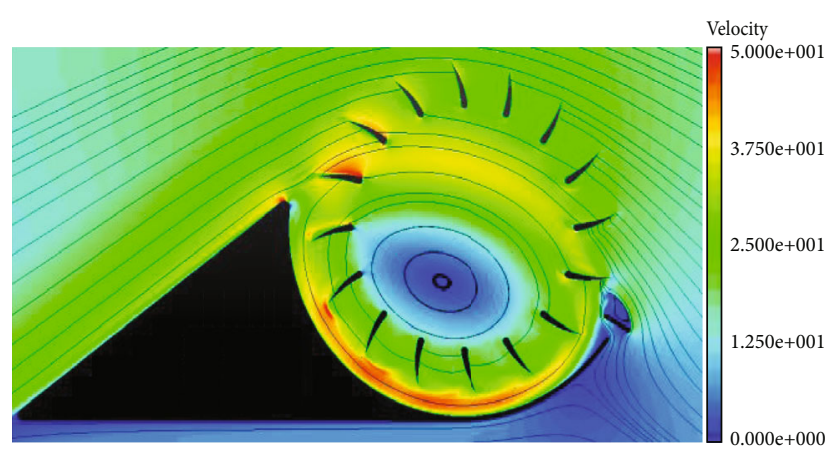

(b) Single winglet

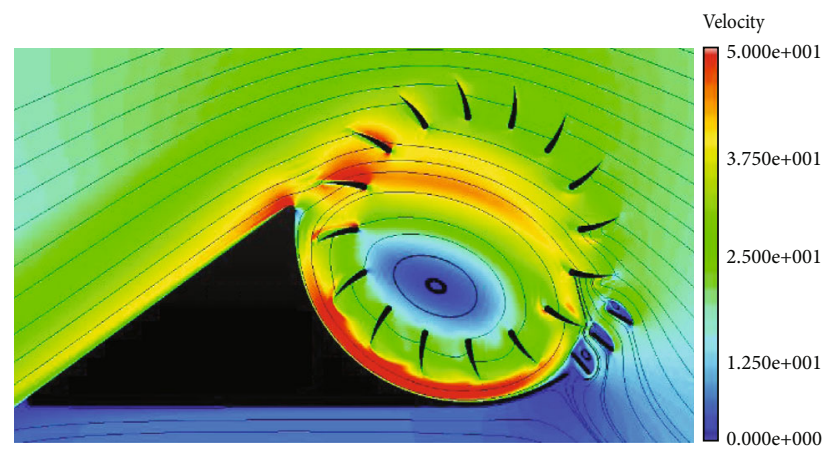

(d) Triple winglet

FIGURE 20: Velocity nephograms and streamlines of different number leading edge winglets (installation angle $=90^{\circ}, v=12 \mathrm{~m} / \mathrm{s}, \alpha=0^{\circ}$ ).

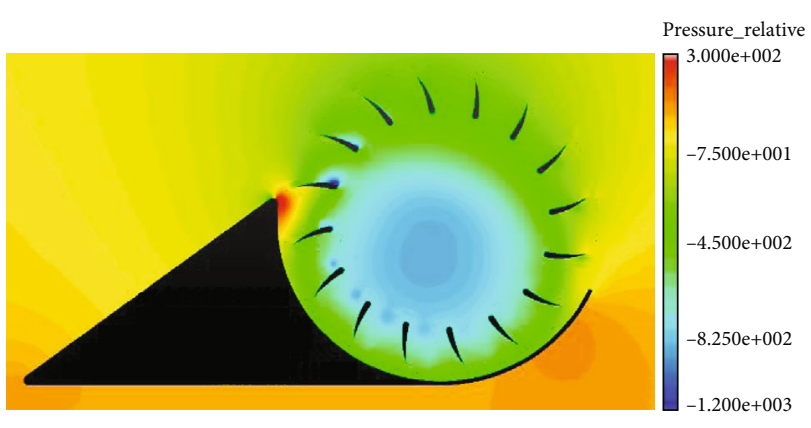

(a) No winglet

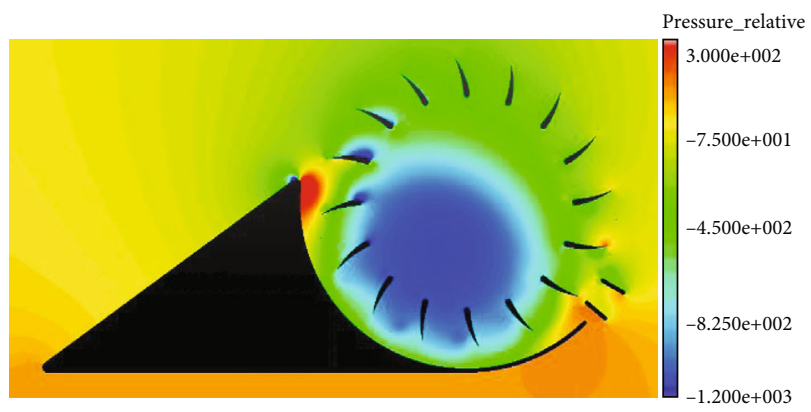

(c) Double winglet

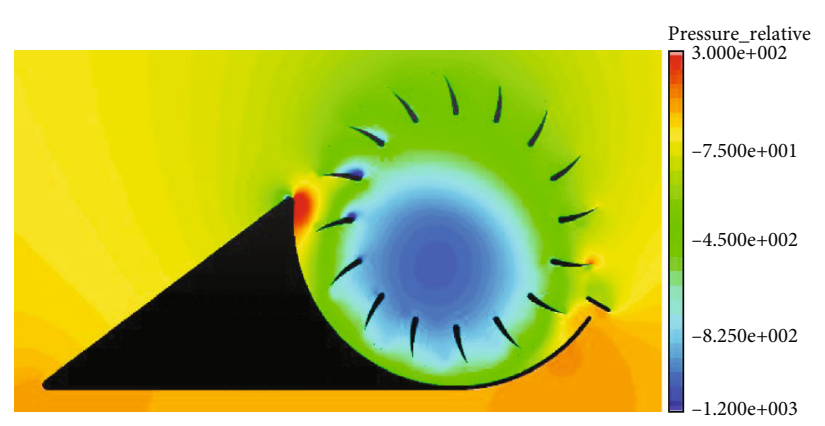

(b) Single winglet

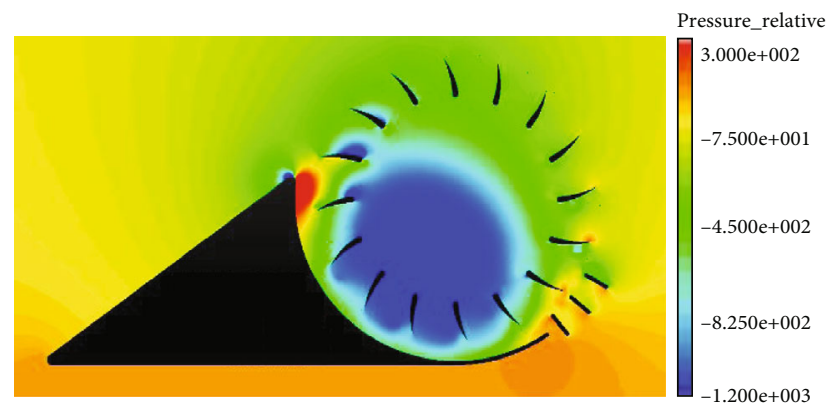

(d) Triple winglet

Figure 21: Pressure nephogram of different number leading edge winglet (Installation angle $=90^{\circ}, \mathrm{v}=12 \mathrm{~m} / \mathrm{s}, \alpha=0^{\circ}$ ). 


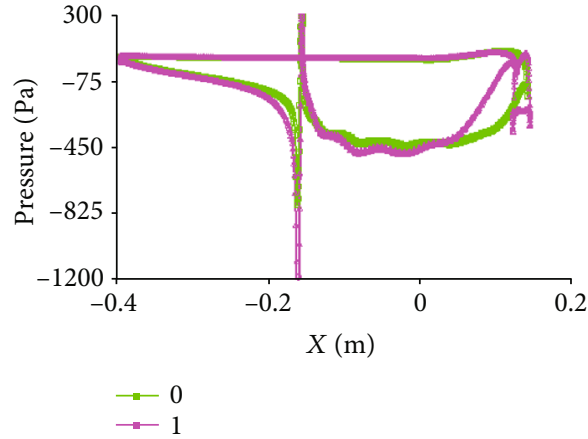

(a) No winglet and single winglet

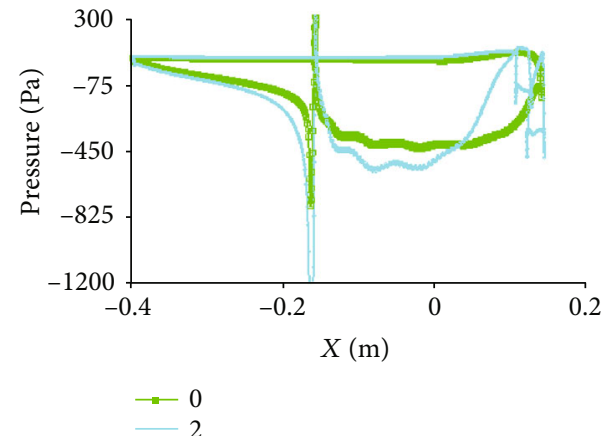

(b) No winglet and double winglet

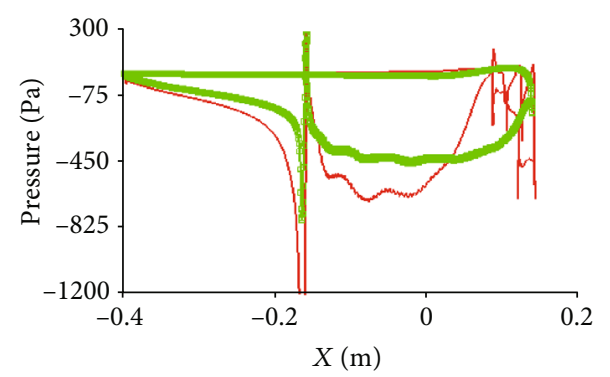

$\longrightarrow 0$

(c) No winglet and triple winglet

FIGURE 22: Static pressure distribution curves of leading edge winglets with different number of blades (installation angle $=90^{\circ}, v=12 \mathrm{~m} / \mathrm{s}$, $\left.\alpha=0^{\circ}\right)$.

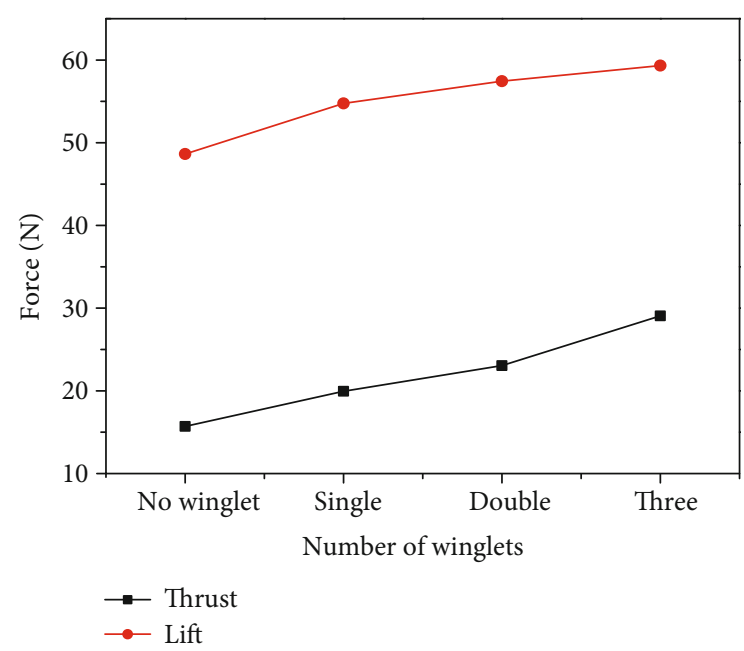

FIGURE 23: The lift/thrust relationship of different number of leading edge winglets.

thrust change is gradually increasing. When triple leading edge winglets are used, the lift and thrust reach the maximum, and the relative change of thrust is greater than that of lift. Table 3 shows the aerodynamic increment comparison of different pieces of winglets with no winglet. Therefore, installing triple leading-edge winglets on the leading edge of the Fan-wing can effectively control the lift and thrust of
TABle 3: Aerodynamic increment comparison of different pieces of winglets with no winglet.

\begin{tabular}{lcc}
\hline Number of winglets & Thrust increment (\%) & Lift increment (\%) \\
\hline Single & 27.09278 & 12.57576 \\
Double & 46.84027 & 18.10627 \\
Triple & 85.10902 & 21.99019 \\
\hline
\end{tabular}

the fan, which also proves that the scheme of installing movable winglets on the leading edge of the fan is feasible.

\section{Conclusion and Discussion}

In this paper, numerical simulation has been used to analyze the effects of deflection angles of small wings with different numbers of blades on the aerodynamic characteristics of the wings. The following conclusions are drawn:

(1) The single leading edge winglet has great influence on the thrust of the Fan-wing, but little influence on the lift. When the deflection angle of the double leading edge winglet changes, the variation of lift and thrust was similar to that of the single leading edge winglet, but the variation of lift and thrust of the double leading edge winglet was larger, which was mainly due to the larger change of the opening angle of the double leading edge winglet. The lift and thrust change of 
triple leading edge winglets to the Fan-wing was larger than that of double leading edge winglets, and the change of thrust relative lift was larger.

(2) Installation of leading edge winglets can change the lift and thrust of the Fan-wing. With the increase of the number of small leading edge winglet, the leading edge opening angle of the Fan-wing can be effectively increased. It was feasible to install movable leading edge winglets on the Fan-wing to change and control the aerodynamic force of the Fan-wing.

\section{Data Availability}

The data used to support the findings of this study are available from the corresponding authors upon request.

\section{Conflicts of Interest}

The authors declare that there is no conflict of interest regarding the publication of this paper.

\section{Acknowledgments}

This project was funded by the China Postdoctoral Science Foundation (Grant No. 2018M642241), Rotor Aerodynamics Key Laboratory (Grant No. RAL20190203), and National Key Laboratory of Rotorcraft Aeromechanics (Grant No. 61422201180510).

\section{References}

[1] K. U. Kogler, Fanwing-experimental evaluation of an ovel lift \& propulsion device. MEng.thesis, Aeronautical Engineering Department, Imperial College, London, 2002.

[2] P. Peebles, "Aerodynamic lift generating device," 2003, US Patent 527229.

[3] G. R. Seyfang, "Fanwing-developments and applications," in 28th Congress of International Council of the Aeronautical Sciences, pp. 1-9, Brisbane, 2012.

[4] G. R. Seyfang, "Recent developments of the Fan-wing aircraft," in The International Conference of the European Aerospace Socoeties, pp. 1-7, Venice, 2011.

[5] S. Foreshaw, Wind Tunnel Investigation of the New fan-Wing Design, Imperial College, London, 1999.

[6] H. S. Bayindir and P. Guillermo, "Analysis of the flow field around the wing section of a FanWing aircraft under various flow conditions," in 53rd AIAA Aerospace Sciences Meeting, Kissimmee, Florida, January 2015.

[7] S. Askari and M. H. Shojaeefard, "Numerical simulation of flow over an airfoil with a cross flow fan as a lift generating member in a new aircraft model," Aircraft Engineering and Aerospace Technology, vol. 81, no. 1, pp. 59-64, 2009.

[8] S. Askari, M. H. Shojaeefard, and K. Goudarzi, "Experimental study of stall in an airfoil with forced airflow provided by an integrated cross-flow fan," Proceedings of the Institution of Mechanical Engineers, Part G: Journal of Aerospace Engineering, vol. 225, no. 1, pp. 97-104, 2011.

[9] S. Askari and M. H. Shojaeefard, "Experimental and numerical study of an airfoil in combination with a cross flow fan," Proceedings of the Institution of Mechanical Engineers, Part
G: Journal of Aerospace Engineering, vol. 227, no. 7, pp. 1173-1187, 2013.

[10] S. Askari and M. H. Shojaeefard, "Shape optimization of the airfoil comprising a cross flow fan," Aircraft Engineering and Aerospace Technology, vol. 81, no. 5, pp. 407-415, 2009.

[11] D. Duddempudi, Y. Yao, D. Edmondson, J. Yao, and A. Curley, "Computational study of flow over generic fanwing airfoil," Aircraft Engineering and Aerospace Technology, vol. 79, no. 3, pp. 238-244, 2007.

[12] T. Q. Dang and P. R. Bushnell, "Aerodynamics of cross-flow fans and their application to aircraft propulsion and flow control," Progress in Aerospace Sciences, vol. 45, no. 1-3, pp. 1-29, 2009.

[13] J. D. Kummer and T. Q. Dang, "High-Lift propulsive Airfoil with integrated Crossflow fan," Journal of Aircraft, vol. 43, no. 4, pp. 1059-1068, 2006.

[14] D. Siliang, T. Zhengfei, X. Pei, and J. Mengjiang, "Study on helicopter antitorque device based on cross-flow fan technology," International Journal of Aerospace Engineering, vol. 2016, Article ID 5396876, 12 pages, 2016.

[15] D. Siliang and T. Zhengfei, "The aerodynamic behavioral study of tandem fan wing configuration," International Journal of Aerospace Engineering, vol. 2018, Article ID 1594570, 14 pages, 2018.

[16] D. Siliang, Z. Qijun, and W. Bo, "Research on distributed jet blowing wing based on the principle of Fan-wing vortexinduced lift and thrust," International Journal of Aerospace Engineering, vol. 2019, Article ID 7561856, 11 pages, 2019.

[17] D. Siliang, L. Zhiminf, and T. Zhengfei, "Numerical simulation research on the boundary control method of the fanwing' $s$ airfoil," Acta aeronautica et Astronautica Sinica, vol. 37, no. 6, pp. 1783-1791, 2016.

[18] D. Siliang, T. Rong Pei, and T. Zhengfei, "Experimental study on aerodynamic characteristics of fanwing," Journal of Nanjing University of Aeronautics \& Astronautics, vol. 49, no. 3, pp. 403-410, 2017.

[19] R. P. Tang, Aerodynamic experimental research on fan-wing, Nanjing University of Aeronautics and Astronautics, Nanjing, 2014. 